Draft Version November 13, 2018

Preprint typeset using IATEX style emulateapj v. 6/22/04

\title{
GRAVITATIONAL SLINGSHOT OF YOUNG MASSIVE STARS IN ORION
}

\author{
Sourav ChatterjeE $^{1}$ and Jonathan C. Tan ${ }^{1,2}$ \\ (Dated:) \\ Draft version November 13, 2018
}

\begin{abstract}
The Orion Nebula Cluster (ONC) is the nearest region of massive star formation and thus a crucial testing ground for theoretical models. Of particular interest amongst the ONC's $\sim 1000$ members are: $\theta^{1}$ Ori $\mathrm{C}$, the most massive binary in the cluster with stars of masses 38 and $9 \mathrm{M}_{\odot}$ (Kraus et al. 2009 ); the Becklin-Neugebauer $(\mathrm{BN})$ object, a $30 \mathrm{~km} \mathrm{~s}^{-1}$ runaway star of $\sim 8 \mathrm{M}_{\odot}$ (Tan 2004); and the Kleinmann-Low (KL) nebula protostar, a highly-obscured, $\sim 15 \mathrm{M}_{\odot}$ object still accreting gas while also driving a powerful, apparently "explosive" outflow (Allen \& Burton 1993). The unusual behavior of $\mathrm{BN}$ and $\mathrm{KL}$ is much debated: How did $\mathrm{BN}$ acquire its high velocity? How is this related to massive star formation in the KL nebula? Here we report the results of a systematic survey using $\sim 10^{7}$ numerical experiments of gravitational interactions of the $\theta^{1} \mathrm{C}$ and $\mathrm{BN}$ stars. We show that dynamical ejection of BN from this triple system at its observed velocity leaves behind a binary with total energy and eccentricity matching those observed for $\theta^{1} \mathrm{C}$. Five other observed properties of $\theta^{1} \mathrm{C}$ are also consistent with it having ejected $\mathrm{BN}$ and altogether we estimate there is only a $\lesssim 10^{-5}$ probability that $\theta^{1} \mathrm{C}$ has these properties by chance. We conclude that $\mathrm{BN}$ was dynamically ejected from the $\theta^{1} \mathrm{C}$ system about 4,500 years ago. BN has then plowed through the KL massive-star-forming core within the last 1,000 years causing its recently-enhanced accretion and outflow activity.

Subject headings: binaries: general - Methods: numerical - Scattering - Stars: individual: BecklinNeugebauer object, $\theta^{1} \mathrm{C}$ - Stars: kinematics and dynamics
\end{abstract}

\section{INTRODUCTION}

Massive stars impact many areas of astrophysics. In most galactic environments they dominate the radiative, mechanical and chemical feedback on the interstellar medium, thus regulating the evolution of galaxies. Many low-mass stars form in clusters near massive stars, and their protoplanetary disks can be affected by this feedback also. There is some evidence that our own solar system was influenced in this way (e.g., Tachibana \& Huss 2003; Adams 2010). Despite this importance, there is no consensus on the basic formation mechanism of massive stars. Theories range from scaled-up versions of low-mass star formation (McKee \& Tan 2003), to competitive Bondi-Hoyle accretion at the center of forming star clusters (Bonnell et al. 2001; Wang et al. 2010), to stellar collisions (Bonnell et al. 1998). The Orion Nebula Cluster (ONC) is the nearest region of massive star formation and thus a crucial testing ground for theoretical models.

Of particular interest amongst the ONC's $\sim 1000$ members in this regard are: $\theta^{1}$ Ori $\mathrm{C}$, the most massive binary in the cluster with stars of masses $\approx 38$ and $9 \mathrm{M}_{\odot}$ (Kraus et al. 2009); the Becklin-Neugebauer (BN) object, a $30 \mathrm{~km} \mathrm{~s}^{-1}$ runaway star of $\approx 8 M_{\odot}$ (Tan 2004); and the Kleinmann-Low (KL) nebula protostar, a highlyobscured, about $15 \mathrm{M}_{\odot}$ object still accreting gas while also driving a powerful, apparently "explosive" outflow (Allen \& Burton 1993). The unusual behavior of BN and $\mathrm{KL}$ is much debated bearing implications towards

\footnotetext{
Electronic address: s.chatterjee@astro.ufl.edu

Electronic address: jt@astro.ufl.edu

1 Department of Astronomy, University of Florida, Gainesville, FL 32611.

2 Department of Physics, University of Florida, Gainesville, FL 32611 .
}

massive-star formation theories: How did BN acquire its high velocity? How is this related to massive star formation in the KL nebula?

$\mathrm{BN}$, like KL, is heavily obscured by dust so its luminosity of $\sim(5 \pm 3) \times 10^{3} \mathrm{~L}_{\odot}$ mostly emerges in the infrared (Gezari et al. 1998). The above luminosity constrains BN's mass to be $m_{\mathrm{BN}} \simeq 9.3 \pm 2.0 \mathrm{M}_{\odot}$, assuming it is on the zero age main sequence (Tan 2004). For this estimate and throughout the paper we have adopted $414 \pm 7 \mathrm{pc}$ for the distance to the cluster (Menten et al. 2007). Astrometry based on $\mathrm{mm}$ and radio observations indicate that BN is a runaway star (Plambeck et al. 1995; Tan 2004), with some recent measurements of its motion in the ONC frame of $\mu_{\mathrm{BN}}=13.2 \pm 1.1$ mas yr $^{-1}$ towards P.A.BN $=-27^{\circ} .5 \pm 4^{\circ}$ (Gómez et al. 2008) and $\mu_{\mathrm{BN}}=$ $13.4 \pm 1.1$ mas yr $^{-1}$ towards P.A.BN $=-18^{\circ} .8 \pm 4.6^{\circ}$ (Goddi et al. 2011) (Figure 1). This corresponds to a velocity $v_{2 \mathrm{D}, \mathrm{BN}}=25.9 \pm 2.2 \mathrm{~km} \mathrm{~s}^{-1}$ (Gómez et al. 2008). BN has an observed radial (LSR) velocity of $+21 \pm \sim 1 \mathrm{kms}^{-1}$ (Scoville et al. 1983), while the ONC mean is $+8.0 \mathrm{kms}^{-1}$ (based on a mean heliocentric velocity of about 1000 ONC stars of $+26.1 \mathrm{~km} \mathrm{~s}^{-1}$; Fürész et al. 2008). Including this $+13 \pm \sim 1 \mathrm{~km} \mathrm{~s}^{-1}$ radial velocity with respect to the ONC mean, the $3 \mathrm{D}$ ONC-frame velocity of $\mathrm{BN}$ is $v_{3 \mathrm{D}, \mathrm{BN}}=29 \pm 3 \mathrm{~km} \mathrm{~s}^{-1}$. This is much greater than the velocity dispersion of ONC stars, variously inferred to be $\sigma_{3 \mathrm{D}}=2.4 \mathrm{~km} \mathrm{~s}^{-1}$ based on the proper motions of $\sim 50$ bright $(V \lesssim 12.5)$ stars within $30^{\prime}$ of the ONC center (van Altena et al. 1988), $\sigma_{3 \mathrm{D}}=3.8 \mathrm{~km} \mathrm{~s}^{-1}$ based on proper motions of $\sim 900$ fainter stars within $15^{\prime}$ of the ONC center (Jones \& Walker 1988), and $\sigma_{3 \mathrm{D}}=5.4 \mathrm{~km} \mathrm{~s}^{-1}$ based on radial velocity measurements (potentially affected by motion induced by binarity) of 1100 stars within $\sim 60^{\prime}$ of the ONC center (Fürész et al. 2008). Thus there is little doubt that BN is a runaway star, which formed and was 
then accelerated in the ONC.

Supernova explosion of one member of a binary can lead to the other being ejected at high speeds (Zwicky 1957 ). The ONC is too young (most stars are $<3$ Myr old; Da Rio et al. 2010) for a supernova to have occurred. Nor is there any evidence for a recent supernova. Alternatively, runaway stars can be produced via dynamical ejection - a gravitational slingshot — from a triple or higher multiple system (Poveda et al. 1967; Hut \& Bahcall 1983; Gies \& Bolton 1986), in which the lowest mass member tends to be ejected. Indeed, such dynamical ejection of stars naturally happen in dense and young clusters (e.g., Gualandris et al. 2004; Fujii \& Portegies Zwart 2011). Similar ejections have also been discussed in the context of some other ONC stars (Gualandris et al. 2004). Thus BN, having formed in the ONC, should have been accelerated via dynamical ejection. The predictions of this scenario are very specific: somewhere along BN's past trajectory should be a massive binary (or higher order multiple), with two components likely more massive than $\mathrm{BN}$, recoiling in the opposite direction, and, as we shall see, with specific orbital properties.

Two scenarios for the dynamical ejection of BN have been proposed. (1) Ejection from the $\theta^{1} \mathrm{C}$ binary (Tan 2004): In this scenario the BN star is ejected via a strong gravitational scattering interaction (e.g., Hut \& Bahcall 1983; Gvaramadze \& Gualandris 2011) and later plows, by chance, through the KL star-forming core to drive tidally-enhanced accretion and thus outflow activity. If so, a model of formation of the KL massive protostar via an ordered collapse of a gas core to a central disk(McKee \& Tan 2003), similar to how low-mass stars are thought to form, is still broadly applicable, though subject to the tidal perturbation from BN's fly-by. (2) Ejection from the KL (source I) protostar (Bally \& Zinnecker 2005; Gómez et al. 2008): Here it is proposed that the KL outflow is related to the disintegration of a forming triple system, which ejected BN and produced a binary suggested to be the radio source I (Menten \& Reid 1995). This binary has recoiled southwards from the original formation site and is now hidden, by chance, behind or in the dense gas core near the center of the KL nebula. In this scenario the gas from the original formation site, like the stars, has also been expelled in this event to form the outflow, and the core that formed these massive stars has been destroyed. If true, this is a very different formation process, and would indicate that chaotic gravitational interactions between multiple protostars followed by complete ejection of both stars and gas are intrinsic features of massive star formation (Bally \& Zinnecker 2005; Bally et al. 2011), at least in this case in Orion.

Figure 1 shows a near-IR image of the central region of the $\mathrm{ONC}$, including $\mathrm{BN}$, the $\mathrm{KL}$ protostar (marked by radio source $I$ ) and the famous Trapezium stars, of which $\theta^{1} \mathrm{C}$ is the brightest. The past trajectory of $\mathrm{BN}$ is indicated based on its present motion and assuming no acceleration. It goes near KL and the Trapezium stars. The high obscuration to KL means that there is little direct constraint on the properties of the $\operatorname{star}(\mathrm{s})$ : for example there is no evidence that it is even a binary. In contrast, the properties of $\theta^{1} \mathrm{C}$ have been measured much more precisely (Kraus et al. 2009) and so the scenario of ejection of BN via a binary-single strong scattering can be tested much more rigorously and is the goal of this study.

In $\S 2$ we summarize our methods and numerical calculations. In $\S 3$ we present our key results, which show that $\theta^{1} \mathrm{C}$ has orbital properties expected if it ejected $\mathrm{BN}$. In $\S 4$ we estimate the probability that $\theta^{1} \mathrm{C}$ has not been responsible for BN's ejection and has these and other observed properties simply by chance. We summarize and conclude in $\S 5$.

\section{METHODS}

We investigate the scenario of ejection of $\mathrm{BN}$ from the $\theta^{1} \mathrm{C}$ binary by carrying out calculations of gravitational scattering between the three stars. We adopt the central values of the observed masses of the $\theta^{1} \mathrm{C}$ binary members (Kraus et al. 2009), $m_{\theta^{1} \mathrm{C}_{1}}=38.2 \pm 3.6 \mathrm{M}_{\odot}$ and $m_{\theta^{1} \mathrm{C}_{2}}=$ $8.8 \pm 1.7 \mathrm{M}_{\odot}$. For BN we adopt the central value of the mass estimate $m_{\mathrm{BN}}=8.2 \pm 2.8 \mathrm{M}_{\odot}$, which is based on the observed cluster-frame proper motion of $\theta^{1} \mathrm{C}$ of $\mu_{\theta^{1} \mathrm{C}}=$ $2.3 \pm 0.2 \mathrm{mas} \mathrm{yr}^{-1}$ (van Altena et al. 1988), i.e., $v_{2 \mathrm{D}, \theta^{1} \mathrm{C}}=$ $4.5 \pm 0.4 \mathrm{~km} \mathrm{~s}^{-1}$, and assuming it is due to recoil from ejecting BN. Note that this mass is consistent with that inferred from the luminosity of BN, discussed above. The error range includes an assumed pre-ejection motion of the center of mass of the 3 stars along the ejection axis

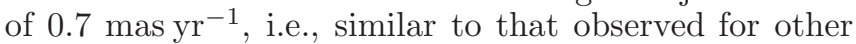
bright ONC stars (van Altena et al. 1988).

We carry out a systematic investigation of the three possible strong scattering interactions between a binary and a single star. Depending on the initial perturber and the binary members these interactions can be divided into three types. Type 1 - BN star is the perturber: Here $\mathrm{BN}$ is initially a single star that interacts with $\theta^{1} \mathrm{C}$ members $\theta^{1} \mathrm{C}_{1}$ and $\theta^{1} \mathrm{C}_{2}$. We denote this initial configuration as $\left[\theta^{1} \mathrm{C}_{1}, \theta^{1} \mathrm{C}_{2}\right] \mathrm{BN}$. The square brackets denote a bound binary and the star outside the brackets is a single stellar perturber. Type $2-\theta^{1} \mathrm{C}_{2}$ is the perturber: This can be denoted as $\left[\theta^{1} \mathrm{C}_{1}, \mathrm{BN}\right] \theta^{1} \mathrm{C}_{2}$. Type $3-\theta^{1} \mathrm{C}_{1}$ is the perturber: This can be denoted as $\left[\theta^{1} \mathrm{C}_{2}, \mathrm{BN}\right]$ $\theta^{1} \mathrm{C}_{1}$.

Out of all outcomes of our numerical experiments we focus on the ones that could lead to ejection of BN. Case 1 - BN fly-by: This is a Type 1 interaction and the outcome of interest is "preservation" where the initial binary members remain unchanged and BN flies by after interaction with the initial binary. We write this interaction as $\left[\theta^{1} \mathrm{C}_{1}, \theta^{1} \mathrm{C}_{2}\right] \mathrm{BN} \longrightarrow\left[\theta^{1} \mathrm{C}_{1}, \theta^{1} \mathrm{C}_{2}\right] \mathrm{BN}$. The arrow points from the initial to the final configuration. Case 2 - ejection of BN from a binary via exchange with $\theta^{1} \mathrm{C}_{2}$ : $\left[\theta^{1} \mathrm{C}_{1}, \mathrm{BN}\right] \theta^{1} \mathrm{C}_{2} \longrightarrow\left[\theta^{1} \mathrm{C}_{1}, \theta^{1} \mathrm{C}_{2}\right] \mathrm{BN}$. Case 3 - ejection of $\mathrm{BN}$ from a binary via exchange with $\theta^{1} \mathrm{C}_{1}$ : $\left[\theta^{1} \mathrm{C}_{2}, \mathrm{BN}\right] \theta^{1} \mathrm{C}_{1} \longrightarrow\left[\theta^{1} \mathrm{C}_{1}, \theta^{1} \mathrm{C}_{2}\right] \mathrm{BN}$. The "Types" and "Cases" are different in the fact that for the Types we only take into account the initial conditions and allow all outcomes, whereas, the Cases are more restrictive and only considers the outcomes where $\mathrm{BN}$ is ejected leaving behind a bound binary.

There are 7 parameters that describe the initial conditions of each binary-single star interaction and since the 3-body problem is chaotic we must sample over the expected distributions of these parameters: (1) Eccentricity of the initial binary, $e_{i}$. For each case we investigate two extreme distributions: (A) Circular, $e_{i}=0$, for all systems, which may be expected if the binaries have recently formed from a gas disk that has damped out 


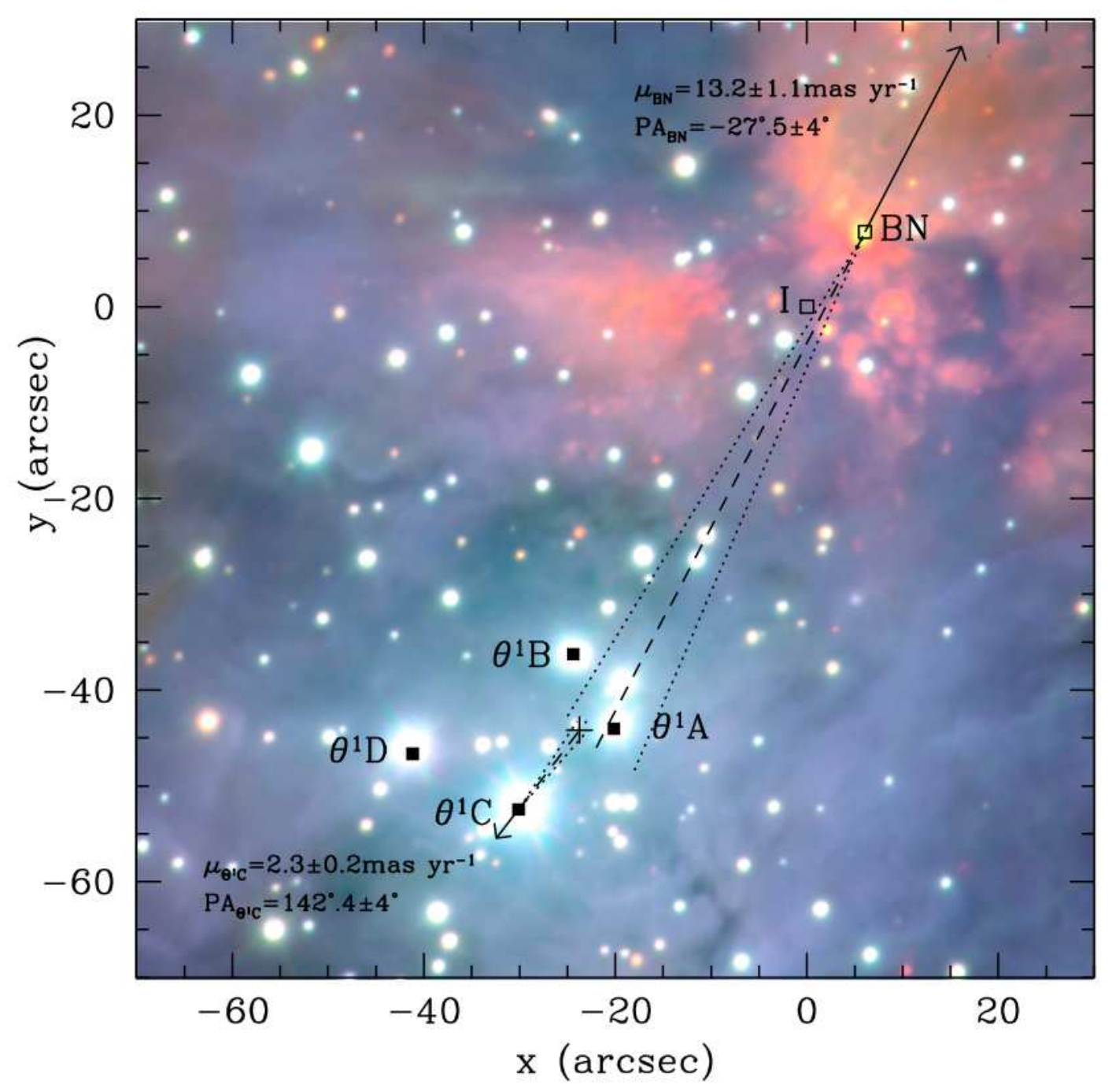

FIG. 1.- Near-infrared (J,H,K) image of the central region of the Orion Nebula Cluster (McCaughrean et al. 2002) with cluster frame proper motions of BN (Gómez et al. 2008; Tan 2008) and $\theta^{1} \mathrm{C}$ (van Altena et al. 1988) indicated by arrows proportional to the size of the motion. The coordinates are relative to the position of source $I(\alpha(\mathrm{J} 2000)=053514.5141, \delta(\mathrm{J} 2000)=-052230.556)(\mathrm{Gómez}$ et al. 2008). Under the simplifying assumption of no acceleration, these motions are traced back with dashed lines (dotted lines indicate $1 \sigma$ uncertainties) to a common origin about 4,500 years ago, shown by the cross at $(-23.72,-44.16)$. The positions of the other Trapezium stars and the Kleinmann-Low massive protostar, source $I$, are also indicated.

noncircular motions; (B) Thermal (Heggie \& Hut 2003), $d F_{b} / d e_{i}=2 e_{i}$, where $F_{b}$ is the fraction of the binary population. This is an extreme scenario that would result if binaries have had time to thermalize via stellar interactions with other cluster stars. The actual situation for ONC binaries should be between these limits. (2) Semimajor axis of the initial binary, $a_{i}$. We assume a flat distribution (Eggleton et al. 1989) $d F_{b} / d \log a_{i}=0.208$ from $0.1 \mathrm{AU}$ (approximately the limit resulting from physical contact) to $6300 \mathrm{AU}$ (the hard-soft boundary beyond which binaries are expected to be disrupted by interactions with other cluster stars; Heggie \& Hut 2003). (3) Initial impact parameter, $b_{i}$. For each Case and each sampling of $a_{i}$ we investigate the full range of impact parameters that can lead to scattering events strong enough to eject BN with its observed high velocity. This is achieved by increasing $b_{i}$ from small values until the regime where all interactions are weak fly-bys incapable of increasing BN's velocity to the observed large value. (4) Initial relative velocity at infinity, $v_{i}$, in the frame of the center of mass of the binary. We assume that the stars have velocities following a Maxwellian distribution with a dispersion of $\sigma_{3 \mathrm{D}}=3 \mathrm{~km} \mathrm{~s}^{-1}$ (Fürész et al. 2008). We have repeated the numerical experiments with $\sigma_{3 \mathrm{D}}=2 \mathrm{~km} \mathrm{~s}^{-1}$, finding qualitatively similar results. (5) The initial angle of the orbital angular momentum vector $\left(\vec{L}_{i}\right)$ of the binary with respect to the velocity vector $\left(\vec{v}_{i}\right)$ of the approaching single star, which is assumed to be randomly oriented. (6) The initial angle between the major axis of the binary orbit and the velocity vector of the approaching single star, which is assumed to be 
randomly oriented. (7) The initial orbital phase of the binary, which is assumed to be random.

We find cross-sections $(\Sigma)$ of the various outcomes of the Cases 1, 2, \& 3 binary-single interactions numerically using the Fewbody software (Fregeau et al. 2004), which uses an order 8 Runge-Kutta integrator, by performing $\sim 10^{7}$ numerical scattering experiments to sample the 7 dimensional parameter space that is needed to describe the possible interactions. This large number of numerical scattering experiments gives us rigorous sampling of all properties in the dynamical scattering problem, including the initial semimajor axis of the binary $\left(a_{i}\right)$, initial eccentricity $\left(e_{i}\right)$, binary orbital phases, initial velocity at infinity $\left(v_{i}\right)$ of the single star, the initial impact parameter of the encounter $\left(b_{i}\right)$, the angle between the initial major axis relative to $\vec{v}_{i}$ and the initial orientation of the binary $\left(\vec{L}_{i} \cdot \vec{v}_{i}\right)$. For example, we sample $b_{i}$ as fine as $10^{-4} b_{0}$, where $b_{0}$ is the impact parameter at infinity that results in a closest approach within $2 a_{i}$. Starting from a small value, $b_{i}$ is sampled with the above-mentioned resolution up to at least $b_{i, \max }=b_{0}$. Within this interval smaller intervals of $b_{i}, \delta b_{i}=10^{-4} b_{0}$ are chosen. The impact parameter is chosen from each of these intervals uniform in the area of the annulus between $b_{i}^{\prime}$ and $b_{i}^{\prime}+\delta b_{i}$. If a particular final outcome of interest or "event" is achieved (in particular BN-Velocity or BN-True events, defined below), then contribution of that event to the total $\Sigma$ is simply $\delta \Sigma=2 \pi b_{i}^{\prime} \delta b_{i}$ (McMillan \& Hut 1996; but see more recently Fregeau et al. 2006). Afterwards, to ascertain that all energetic encounters are sampled, we increase the maximum $b_{i}$ geometrically until $b_{i, \max }=100 b_{0}$. For our case, this large value of $b_{i, \max }$ corresponds to as large a physical distance as the cluster size making sure that all possible energetic encounters are captured in the determination of the cross-sections $\Sigma$.

Using the large ensemble of numerical gravitational scattering experiments we evaluate the $\Sigma$ s for outcomes where $\mathrm{BN}$ is ejected leaving behind $\theta^{1} \mathrm{C}_{1}$ and $\theta^{1} \mathrm{C}_{2}$ in a bound binary (henceforth, "BN-Ejection" events). A subset of the BN-Ejection events where BN is ejected with the observed velocity of $29 \pm 3 \mathrm{~km} \mathrm{~s}^{-1}$ are called "BN-Velocity" events. We do not put any constraints on the binary properties that is left behind for the BN-Velocity events. We further calculate the $\Sigma$ s of a subset of BN-Velocity events where the final binary is left with orbital properties similar to those observed of $\theta^{1} \mathrm{C}$, namely, $a=18.13 \pm 1.28 \mathrm{AU}$, and $e=0.592 \pm 0.07$ (Kraus et al. 2009, henceforth, "BN-True" events). The BN-Ejection events are used to explore the velocity distribution of $\mathrm{BN}$ if it is ejected via a strong binarysingle interaction. BN-Velocity events, a subset of the BN-Ejection events, show us all possible interactions over a range of $a_{i}$ where BN could have an energy compatible with the observed energy. A further subset, the BN-True events, give us stronger constraints and indicates the range of initial binary properties most likely to create the observed $\theta^{1} \mathrm{C}$ binary as well as the runaway BN star.

\section{RESULTS}

In this section we present the key results of our numerical experiments. We start with overall outcomes of all our numerical experiments for all Cases and $e_{i}$ distributions and then increasingly focus our attention towards the observed $\mathrm{BN}-\theta^{1} \mathrm{C}$ system and compare its various properties with those predicted from our simulations.

Figure 2 shows the branching ratios of all outcomes in general from our numerical experiments. A handful of interesting aspects are evident in the branching ratios for the given masses of the 3 stars involved in these interactions.

Disruption (or ionization) of the initial binary happens only when the binary is dynamically soft (Hut \& Bahcall 1983), i.e., the value of the binary binding energy is $\lesssim$ the kinetic energy of the perturber. This is achieved at large $a_{i} \gtrsim 300 \mathrm{AU}$, for Types 1 and 2. For Type 3 ionization can happen at relatively smaller $a_{i} \sim 50$ AU due to the higher mass of the perturber and relatively lower binding energy of the initial binary. Nevertheless, even for Type 3 , branching ratio for ionization becomes comparable or greater than exchange outcomes only at $a_{i} \sim 10^{3} \mathrm{AU}$.

For Types 1 and 2, exchange with the primary is very unlikely, since here the primary is significantly more massive $\left(38.2 \mathrm{M}_{\odot}\right)$ than the secondary $\left(8.8\right.$ and $8.2 \mathrm{M}_{\odot}$ for interactions of Types 1 and 2, respectively). In interaction of Type 3, since the initial binary consists of two stars with comparable masses, both exchanges are almost equally likely. For Type 3 the fraction of exchange outcomes is comparable to the fraction of fly-by events for a large range of $a_{i}$ taking into account sufficiently strong encounters (see $\S 2$ for the value of the maximum impact parameter). The fraction of fly-by outcomes is of course formally infinite since one can always use a sufficiently large impact parameter where nothing but a weak fly-by is the outcome.

Collisional outcomes are comparable with exchanges only for sufficiently small $a_{i}$ values. In interactions of Type 1, preservation is the channel that can produce the observed BN- $\theta^{1} \mathrm{C}$ system. For Type 1 for both $e_{i}$ distributions collisions become important for $a_{i} \lesssim 1 \mathrm{AU}$. For interactions of Types 2 and 3, exchange of the perturber with the primary is the channel that can produce the observed $\mathrm{BN}-\theta^{1} \mathrm{C}$ system. For Type 2 , collisions become comparable with the BN- $\theta^{1} \mathrm{C}$ producing channel for $a_{i} \lesssim$ a few AU. Whereas, for interactions of Type 3, collisions remain comparable to the $\mathrm{BN}-\theta^{1} \mathrm{C}$ producing channel for $a_{i} \lesssim 1 \mathrm{AU}$. In interactions of all Types collisions happen more often for the Thermal $e_{i}$ distribution since for the Thermal $e_{i}$ distribution the pericenter distances for the stars in binary can be much smaller than that for the Circular $e_{i}$ distribution for any given $a_{i}$. We later show (§3.3) that BN-True events happen in a given range of $a_{i}$ for a given interaction Type. For all interaction Types and $e_{i}$ distributions collisions have much lower branching ratios compared to the branching ratios for the $\mathrm{BN}-\theta^{1} \mathrm{C}$ production channels for the ranges of $a_{i}$ where BN-True events can occur.

In the following sections we increasingly focus on outcomes that are similar to the observed $\mathrm{BN}-\theta^{1} \mathrm{C}$ system. We first present results for all events where the BN star is ejected (BN-Ejection, $\S 3.1$ ). Then we present results for all outcomes where the BN star is ejected with a velocity within the observed range of $29 \pm 3 \mathrm{kms}^{-1}$ (BN-Velocity, $\S 3.2)$. We then restrict our attention to only a subset of the BN-Velocity events where the final binary has properties similar to the observed $\theta^{1} \mathrm{C}$ binary (BN-True, $\S 3.3$ 


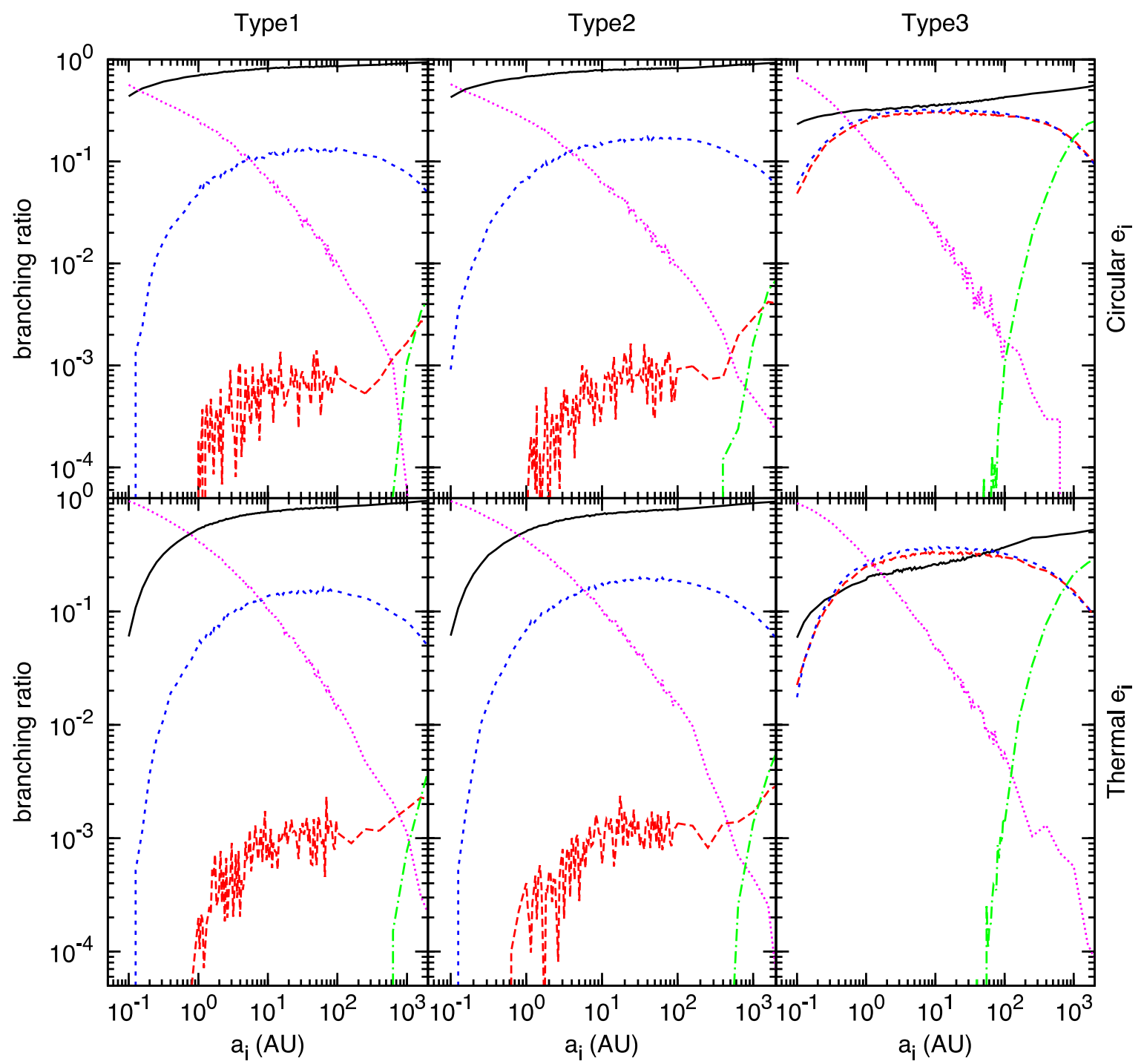

FIG. 2.- Branching ratios for various outcomes in our simulations as a function of $a_{i}$. Solid (black), dotted (magenta), short-dashed (blue), long-dashed (red), and dash-dotted (green) lines denote branching ratios for preservation, collisional outcome, exchange of the perturber with the secondary of the initial binary, exchange of the perturber with the initial primary, and disruption of the binary, respectively. The top and bottom panels are for Circular and Thermal $e_{i}$ distributions, respectively. In both top and bottom panels three panels from left to right denote Types 1,2 , and 3 , respectively.

and $\S 3.4)$.

\subsection{Velocity Distribution of the Ejected BN Star}

Increasing the kinetic energy of BN by about two orders of magnitude compared to the value expected given the ONC's velocity dispersion is at the heart of the problem. Hence, we focus on the velocity distribution of $\mathrm{BN}$ following ejection. In addition, we focus on energy considerations for the scattering problem, especially the ratio of the kinetic energy of BN's ejection to the total energy of the binary left behind.

First we explore given the masses of the three stars in the interaction, and given that $\mathrm{BN}$ is ejected leaving the other stars in a binary, how likely it is for BN to acquire a velocity significantly higher than the velocity dispersion $\left(v_{\sigma}=3 \mathrm{~km} \mathrm{~s}^{-1}\right)$ in the ONC. We calculate the cross-section $\Sigma_{\mathrm{BN}-\text { Ejection }}$ for BN-Ejection events for a given $a_{i}$, for each Cases 1-3, and each $e_{i}$ distribu- tion. The overall cross-section for BN-Ejection events for any $a_{i}$ is calculated by multiplying $\Sigma_{\mathrm{BN}-\text { Ejection }}$ with the probability of finding an initial binary with that $a_{i}$ assuming the semimajor axis distribution for binaries is flat in logarithmic intervals within the physical limits discussed in $\S 2$. Thus, the normalized $\Sigma_{\mathrm{BN}-\text { Ejection }}$ is $\int P\left(a_{i}\right) \Sigma_{\mathrm{BN}-\text { Ejection }}\left(a_{i}, v_{\mathrm{BN}}, e\right) d a_{i}$, where, $P\left(a_{i}\right) d a_{i}=$ $d \log \left(a_{i}\right) / \log (6310 / 0.1)$.

Figure 3 shows the cumulative distribution of $\Sigma_{\mathrm{BN}-\text { Ejection }}\left(v_{\mathrm{BN}}\right)$ as a function of BN's velocity $\left(v_{\mathrm{BN}}\right)$ calculated using $\int_{v_{\mathrm{BN}}=2 v_{\sigma}}^{v} d v_{\mathrm{BN}} \times d \Sigma_{\mathrm{BN}-\text { Ejection }} / d v_{\mathrm{BN}}$. We find that binary-single interactions involving the three stars in question can eject the BN star with velocities that can exceed $v_{\sigma}$ by more than two orders of magnitude. However, the cross-sections for such events reduce as $v_{\mathrm{BN}}$ increases. For both $e_{i}$ distributions Case 3 shows a higher fraction of high-velocity ejection events. This is because in Case 3 the perturber is the most massive 


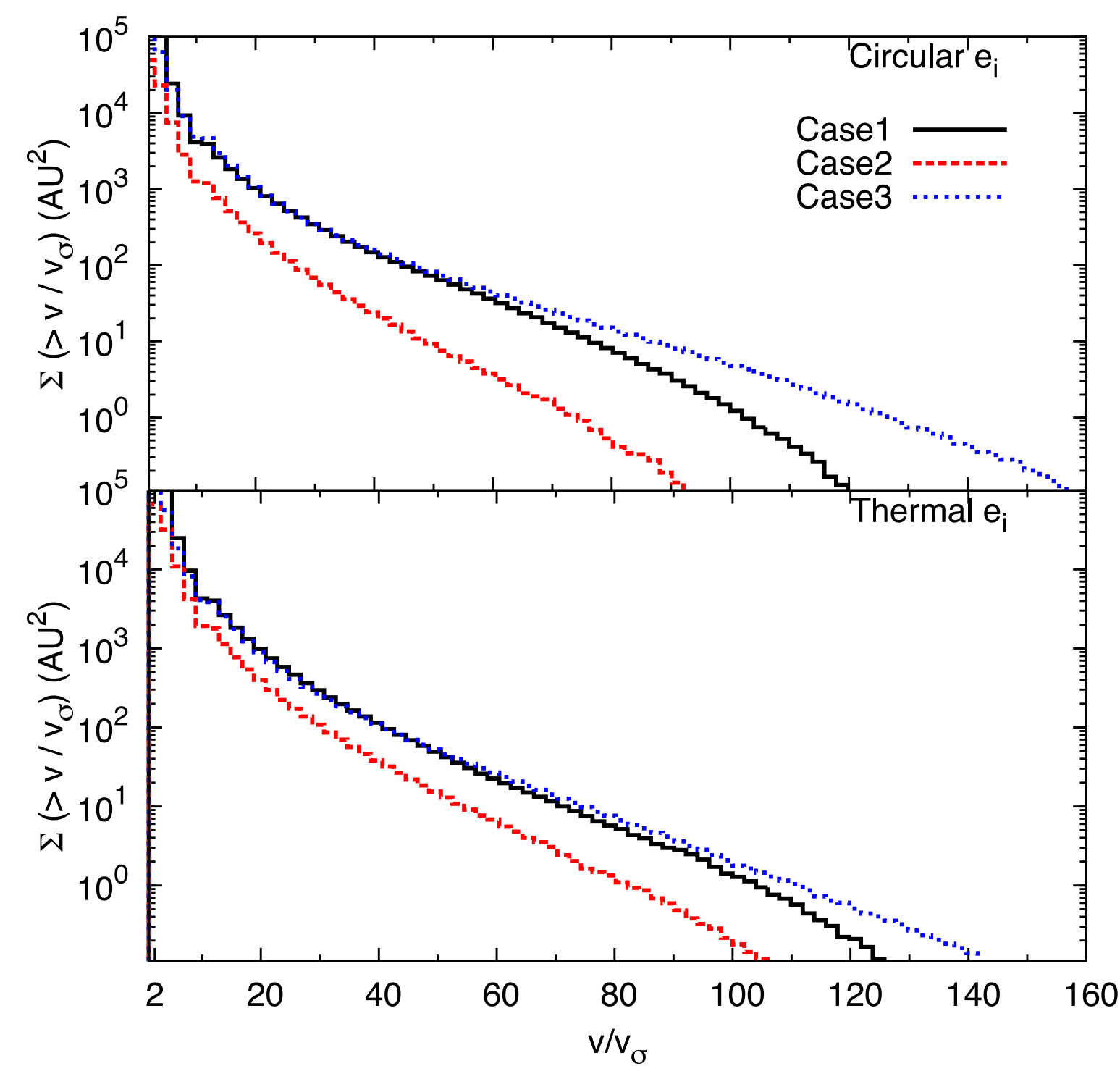

FIG. 3.- Cumulative distribution of $\Sigma$ as a function of the velocity of the ejected star for all cases where BN is ejected. The velocities are given in units of the velocity dispersion $\left(v_{\sigma}=3 \mathrm{kms}^{-1}\right)$. Top panel is for the Circular $e_{i}$ distribution. The bottom panel is the same but for the Thermal $e_{i}$ distribution. The solid (black), dashed (red), and dotted (blue) lines in both panels denote Cases 1, 2, and 3, respectively. For each case the cross-section is calculated using $\int_{v^{\prime}=2 v_{\sigma}}^{v} \int_{a_{i}} P\left(a_{i}\right) \frac{d \Sigma\left(a_{i}, E_{\mathrm{ratio}}, e\right)}{d v^{\prime}} d a_{i} d v^{\prime}$ for all events where BN is ejected. For all cases $P\left(a_{i}\right) d a_{i}=\delta \log \left(a_{i}\right) / \log (6310 / 0.1)$ is used (see text). We use a cut-off for the ejection velocity at $v=2 v_{\sigma}$ since for $v \sim v_{\sigma} \Sigma\left(>v / v_{\sigma}\right)$ becomes very large simply due to distant fly-by interactions in Case 1 . Note that for all cases dynamical interactions can increase the velocity of the ejected star by large factors relative to the velocity dispersion.

star in the triplet, the one that finally becomes the $\theta^{1} \mathrm{C}_{1}$ star. Hence, the total available energy is higher in Case 3 events.

The problem of binary-single scattering can be understood by comparing the kinetic energy and the potential energy of the systems since the outcomes differ qualitatively depending on the relative values of these quantities (e.g., Hut \& Bahcall 1983). We explore if $\mathrm{BN}$ is ejected with $v_{\mathrm{BN}} \geq 2 v_{\sigma}$, then what is the distribution of cross-section for the various Cases and $e_{i^{-}}$ distributions as a function of the $E_{\text {ratio }}$ and the velocity of ejection, $v_{\mathrm{BN}}$. Here, $E_{\text {ratio }} \equiv T_{\text {ejection }} /\left|E_{\text {binary }}\right|$ is the ratio of the final kinetic energy $\left(T_{\text {ejection }}\right)$ of both the single star and the binary star system (based on the motion of its center of mass) to the total energy (gravitational energy plus kinetic energy of orbital motion) of the binary $\left(E_{\text {binary }}\right)$. Figure 4 shows a $2 D$ distribution of the overall $\Sigma_{\mathrm{BN}-\text { Ejection }}$ for any $a_{i}$ as a function of $v_{\mathrm{BN}}$ and $E_{\text {ratio }}$ for all Cases 1-3, and all $e_{i}$ distributions. The highest ejection velocities for the BN star happens for $E_{\text {ratio }}$ between 0.1 and 1 for all Cases. Note that the distributions for Cases 1 and 2 are very similar. This is due to the similarity in masses of $\mathrm{BN}$ and $\theta^{1} \mathrm{C}_{2}$. However, in Case 3 the most massive star is the perturber. In addition, the most massive star exchanges into the final binary increasing the binding energy of the final binary star system. Hence, there is a tail for high $E_{\text {ratio values }}$ where BN can still be ejected with a high velocity. Even for the more energetic Case 3 events, the maximum velocity for ejections are achieved within a narrow range of 
Cose 1: $\left[\theta^{1} \mathrm{C}_{1}, \theta^{1} \mathrm{C}_{2}\right] \mathrm{BN}->\left[\theta^{1} \mathrm{C}_{1}, \theta^{1} \mathrm{C}_{2}\right] \mathrm{BN} \quad \mathrm{Cose2:}\left[\theta^{1} \mathrm{C}_{1}, \mathrm{BN}\right] \theta^{1} \mathrm{C}_{2}->\left[\theta^{1} \mathrm{C}_{1}, \theta^{1} \mathrm{C}_{2}\right] \mathrm{BN} \quad$ Cose3: $\left[\theta^{1} \mathrm{C}_{2}, \mathrm{BN}\right] \theta^{1} \mathrm{C}_{1}->\left[\theta^{1} \mathrm{C}_{1}, \theta^{1} \mathrm{C}_{2}\right] \mathrm{BN}$

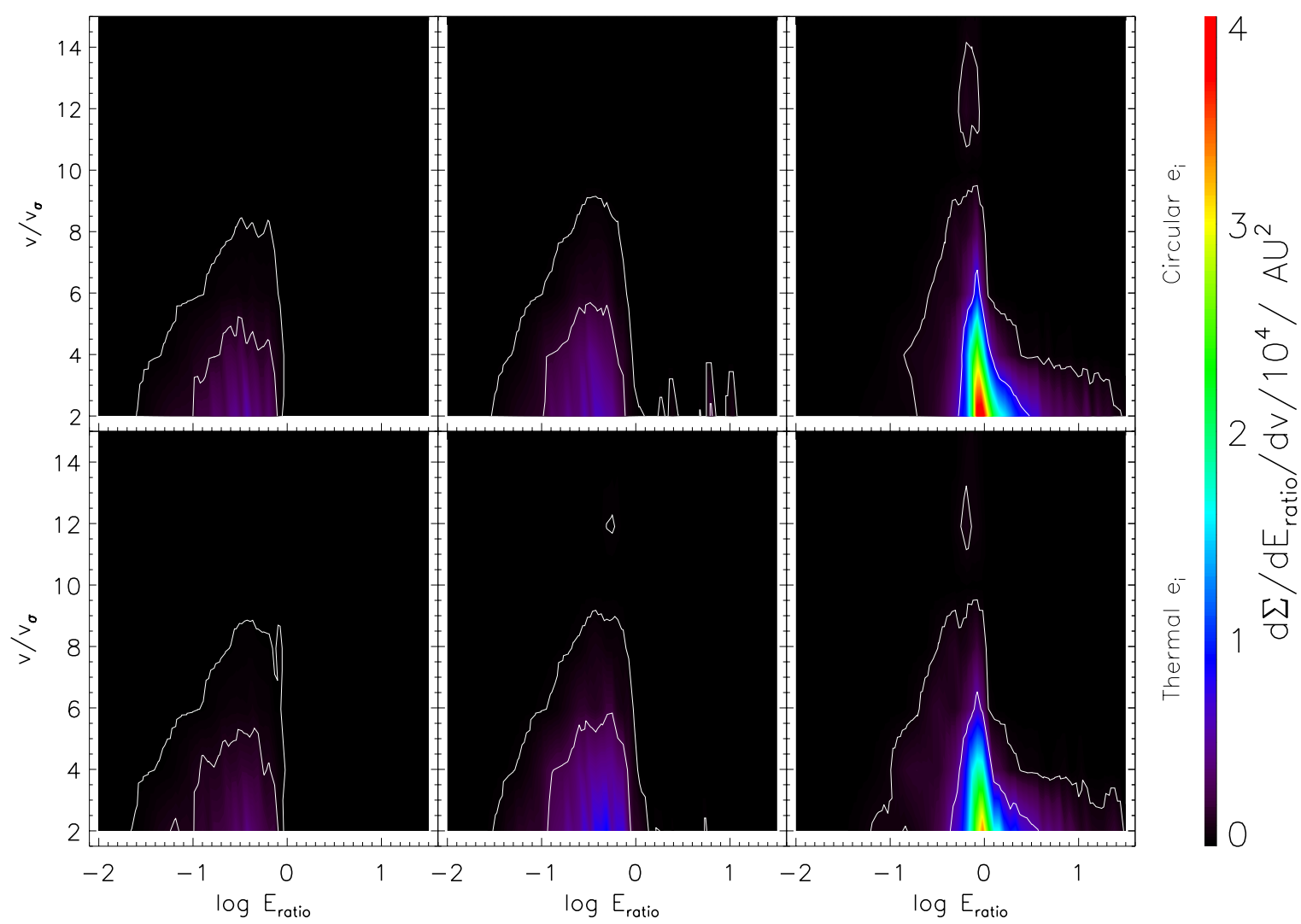

FIG. 4. $-2 \mathrm{D}$ distribution of $\Sigma$ as a function of energy ratio $E_{\text {ratio }} \equiv T_{\text {ejection }} /\left|E_{\text {binary }}\right|$ and velocity $(v)$ of the runaway star BN for all events where $\mathrm{BN}$ is ejected. The top panels are for the Circular $e_{i}$ distributions for Cases 1-3 (left to right, respectively). The bottom panels are for the same, but for the Thermal $e_{i}$ distribution. The colors denote $d \Sigma / d E_{\text {ratio }} / d v$. For each bin the total crosssection is calculated using $\int P\left(a_{i}\right) \Sigma\left(a_{i}, E_{\mathrm{ratio}}, e\right) d a_{i}$ for the events where $\mathrm{BN}$ is ejected satisfying the ranges of $E_{\mathrm{ratio}}$ and $v$ in that bin. $P\left(a_{i}\right) d a_{i}=\delta \log \left(a_{i}\right) / \log (6310 / 0.1)$ is used (see text). For both $e_{i}$ distributions Case3 shows higher values of $E_{\text {ratio }}$ than Cases 1 and 2 . This is because in Case 3 the perturber is a more massive star (the one that will finally become $\theta^{1} \mathrm{C}_{1}$ ). For all Cases the highest velocity increase for the runaway star is between $E_{\text {ratio }}=0.1$ and 1 .

\section{$0.1<E_{\text {ratio }}<1$.}

A high value $(\sim 10)$ of the ratio between the velocities of the incoming single star (expected to be near $v_{\sigma}$ ) and the ejected single star is needed to create runaway stars by definition. This is possible for strong encounters involving a binary and a single star where a fraction of binding energy of the binary is converted into the kinetic energy $(T)$ of the ejected star. If the final outcome is again a binary and the runaway ejected star (the binary members are not required to remain the initial ones, e.g., for Cases 2 and 3) then the kinetic energy of the stars undergoing dynamical ejection, $T_{\text {ejection }}=(1 / 2) m_{\mathrm{BN}} v_{\mathrm{BN}}^{2}+$ $(1 / 2)\left(m_{\theta^{1} \mathrm{C}_{1}}+m_{\theta^{1} \mathrm{C}_{2}}\right) v_{\theta^{1} \mathrm{C}}^{2} \rightarrow(6.9 \pm 2.7) \times 10^{46} \mathrm{erg}+$ $(1.2 \pm 0.5) \times 10^{46} \mathrm{erg}=(8.1 \pm 2.8) \times 10^{46} \mathrm{erg}$ (here $\rightarrow$ indicates the observed values) is expected to be less than the magnitude of the total energy of the resulting binary, $\left|E_{\text {binary }}\right|=G m_{\theta^{1} \mathrm{C}_{1}} m_{\theta^{1} \mathrm{C}_{2}} /(2 a) \rightarrow(16.4 \pm 4.9) \times 10^{46} \mathrm{erg}$. In addition to requiring $E_{\text {ratio }}<1$, one also expects it to achieve a value of order unity, i.e. not too much less than one. For $E_{\text {ratio }} \ll 1$, collisional outcomes dominate (Figure 2; also see e.g., Hut \& Bahcall 1983; Fregeau et al. 2004). On the other hand, for $E_{\text {ratio }} \gg 1$ outcomes with a disruption of the binary dominates creating 3 single stars (Figure 2). The observed value for the $\mathrm{BN}-\theta^{1} \mathrm{C}$ system is $E_{\text {ratio }} \rightarrow 0.49 \pm 0.22$, consistent with it being a result of a binary-single interaction.

\subsection{Kinetic Energy of Ejection and Eccentricity of Binary}

We now focus on the subset of BN-Ejection events (BN-Velocity) where BN is ejected with the observed velocity of $29 \pm 3(\S 2)$ via any one of the Cases $1-3$ (a small strip in the vertical axis in Figure 4$)$. The $\theta^{1} \mathrm{C}$ orbit is eccentric $(e \approx 0.6)$. Indeed, strong encounters are expected to leave behind binaries with generally high eccentricities. We now want to see the $2 D$ distribution of overall cross-section for all BN-Velocity events for any $a_{i}$ as a function of $E_{\text {ratio }}$ and the final eccentricity.

In Figure 5 we plot the 2D distributions of $\Sigma$ for the BN-Velocity events in the $E_{\text {ratio }}$ versus final $e$ plane for Cases 1, 2, \& 3 for both the Circular and Thermal $e_{i}$ distributions. The observed values of the $\mathrm{BN}-\theta^{1} \mathrm{C}$ system are also shown. These overlap within the $1 \sigma$ contours for all cases. The energy of the $\theta^{1} \mathrm{C}$ binary is just what we would expect if it had ejected $\mathrm{BN}$ at the observed velocity. Its high eccentricity, $\approx 0.6$, is also naturally explained by the recent ejection of $\mathrm{BN}$ since during ejection the potential of the system is changing rapidly.

Note that if the $\theta^{1} \mathrm{C}$ binary was unrelated to $\mathrm{BN}$, then 
Case 1: $\left[\theta^{1} \mathrm{C}_{1}, \theta^{1} \mathrm{C}_{2}\right] \mathrm{BN}->\left[\theta^{1} \mathrm{C}_{1}, \theta^{1} \mathrm{C}_{2}\right] \mathrm{BN} \quad$ Case2: $\left[\theta^{1} \mathrm{C}_{1}, \mathrm{BN}\right] \theta^{1} \mathrm{C}_{2}->\left[\theta^{1} \mathrm{C}_{1}, \theta^{1} \mathrm{C}_{2}\right] \mathrm{BN} \quad$ Cose $3:\left[\theta^{1} \mathrm{C}_{2}, \mathrm{BN}\right] \theta^{1} \mathrm{C}_{1}->\left[\theta^{1} \mathrm{C}_{1}, \theta^{1} \mathrm{C}_{2}\right] \mathrm{BN}$

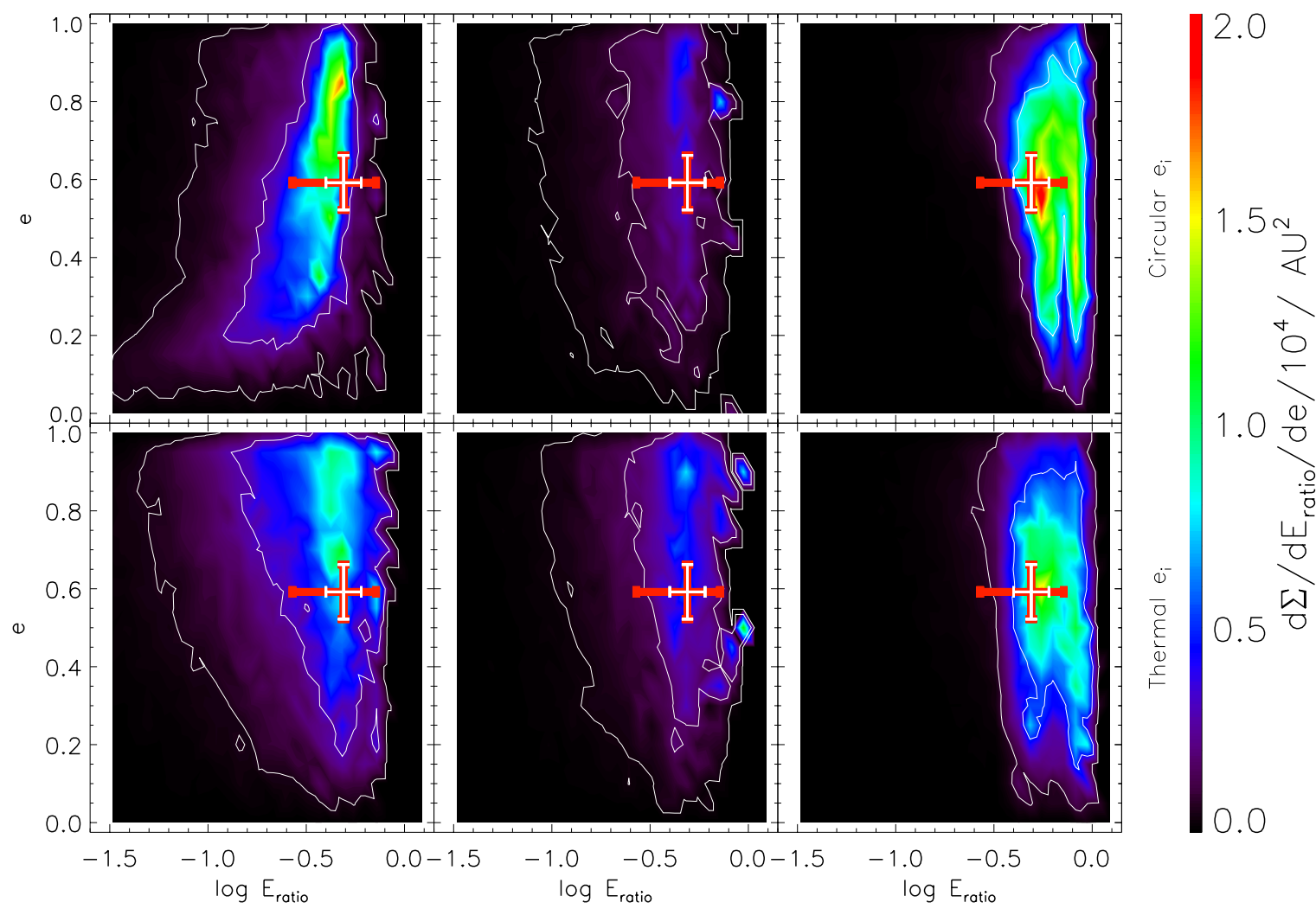

FIG. 5. - 2D distribution of $\Sigma$ as a function of energy ratio $E_{\text {ratio }} \equiv T_{\text {ejection }} /\left|E_{\text {binary }}\right|$ and final eccentricity of the resulting binary $e$ for the BN-Velocity events for all cases and $e_{i}$ distributions. The top panels are for the Circular $e_{i}$ distributions for Cases 1-3 (left to right, respectively). The bottom panels are for the same, but for the Thermal $e_{i}$ distribution. The colors denote $d \Sigma / d E_{\mathrm{ratio}} / d e$. For each bin the total cross-section is calculated using $\int P\left(a_{i}\right) \Sigma\left(a_{i}, E_{\mathrm{ratio}}, e\right) d a_{i}$ for the BN-Velocity events satisfying the ranges of $E_{\mathrm{ratio}}$ and $e$ in that bin. $P\left(a_{i}\right) d a_{i}=\delta \log \left(a_{i}\right) / \log (6310 / 0.1)$ is used (see text). The point and errorbars show the observed BN- $\theta^{1} \mathrm{C}$ system. The larger horizontal errorbars (red) denote the $E_{\text {ratio }}$ errors including contribution from the mass errors of the stars, whereas the shorter (white) errorbars denote the same if the central mass values are chosen and no contribution from mass errors are included (consistent with the numerical experiments). For all cases and all $e_{i}$-distributions the observed system properties lie within the $1 \sigma$ contours of the $\Sigma$-distribution, with Case 3 Circular $e_{i}$ being somewhat more favored (see text).

$E_{\text {ratio }}$ could have values in a large range spanning many orders of magnitude. For example, the range in $a$ for the binary is determined by contact ( $\sim$ a few stellar radii) to the hard-soft boundary in $\mathrm{ONC}(\sim 6000 \mathrm{AU}$ for the velocity dispersion in $\mathrm{ONC}$ ). Thus $E_{\text {binary }}$ for $\theta^{1} \mathrm{C}$ if unrelated to BN's ejection, could be expected to have values anywhere between $\sim 10^{44}$ and $10^{49}$ ergs. It is thus very interesting to find the observed $\mathrm{BN}-\theta^{1} \mathrm{C}$ system with energies so close to the expected energies if they have had a binary-single scattering encounter in the past. The likelihood of this occuring simply by chance rather than by being caused by interaction with BN is explored in $\S 4$.

\subsection{Cross-Sections of BN-Velocity and BN-True Events:}

We evaluate the cross-sections for outcomes where BN is ejected with the observed velocity of $29 \pm 3 \mathrm{~km} \mathrm{~s}^{-1}$ ("BN-Velocity" events). We further calculate the crosssection of a subset of BN-Velocity events where the final binary is left with orbital properties similar to those observed of $\theta^{1} \mathrm{C}$, namely, $a=18.13 \pm 1.28 \mathrm{AU}$, and $e=0.592 \pm 0.07$ (Kraus et al. 2009; "BN-True" events).

For Cases 1, 2, 3 with Circular initial $e_{i}$ distribution, $\Sigma_{\mathrm{BN}-\text { Velocity }}=(2.7,0.94,2.9) \times 10^{3} \mathrm{AU}^{2}$, while for Thermal initial $e_{i}$ distribution, $\Sigma_{\mathrm{BN} \text {-Velocity }}=$ $(2.9,1.4,2.5) \times 10^{3} \mathrm{AU}^{2}$. For Cases 1, 2, 3 with Circular $e_{i}$-distribution, $\Sigma_{\mathrm{BN}-\text { True }}=56,24,105 \mathrm{AU}^{2}$, while for thermal $e_{i}$ distribution, $\Sigma_{\mathrm{BN}-\text { True }}=58,34,84 \mathrm{AU}^{2}$. The three cases have similar cross-sections, with Case 3, i.e. $\left[\theta^{1} \mathrm{C}_{2}, \mathrm{BN}\right] \theta^{1} \mathrm{C}_{1} \longrightarrow\left[\theta^{1} \mathrm{C}_{1}, \theta^{1} \mathrm{C}_{2}\right] \mathrm{BN}$, somewhat more preferred.

The cross-sections for BN-Velocity and BN-True events as a function of $b_{i}$, are shown in Figure 6 . The cross-sections for a given $b_{i}$ and for all explored $a_{i}$ are normalized using the probability, $P\left(a_{i}\right)$, of finding a binary with semimajor axis $a_{i}$, assuming a semimajor axis distribution flat in log intervals. For small values of $b_{i} \lesssim 300 \mathrm{AU}$, the cross-sections grow geometrically as $b_{i}^{2}$ and then decline. This decline, seen in all cases, is simply due to the fact that the interactions are happening at larger and larger impact parameters and at some point no interactions are expected to be strong enough to increase the energy of the ejected star to the observed value of $\mathrm{BN}$. The areas under the histograms are the total cross-sections for the BN-Velocity and BN-True events. Figure 6 also includes a table summarizing the total cross-sections. 


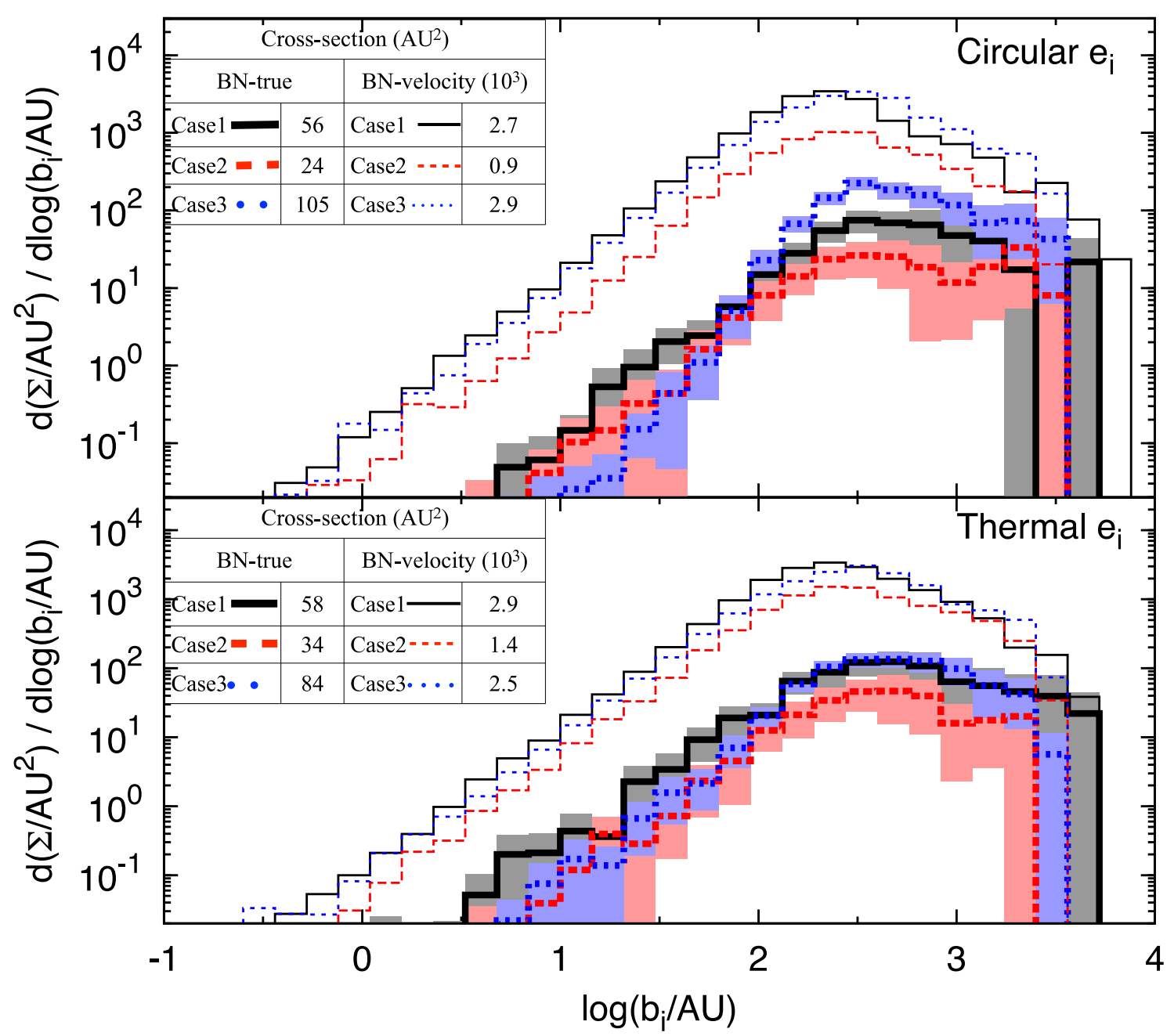

FIG. 6.- Cross-section, $\Sigma$, vs initial impact parameter, $b_{i}$, of interaction. The top and bottom panels show results for the Circular and Thermal $e_{i}$ distributions, respectively. Thin and thick lines represent BN-Velocity and BN-True events, respectively. Black (solid), red (long-dashed), and blue (short-dashed) lines denote Cases 1, 2, and 3, respectively. The power-law increase in cross-section for low $b_{i}$ is simply due to geometry. Most interactions take place within $b_{i} \approx 300 \mathrm{AU}$. However, interactions with $b_{i}$ much larger $\left(\gtrsim 10^{3} \mathrm{AU}\right)$ may still contribute. The color-matched shaded regions around each BN-True histogram denote Poisson errors in estimating $\Sigma$. The inset tables denote the total $\Sigma$ values for BN-Velocity and BN-True events for all cases and $e_{i}$-distributions. All three cases can contribute towards producing the observed $\mathrm{BN}-\theta^{1} \mathrm{C}$ system, however, Case 3 is preferred by a factor of $\sim 2$.

The cross-sections for BN-Velocity and BN-True events as a function of $a_{i}$ are shown in Figure 7 . We find that BN-Velocity events happen via binary-single scattering encounters for a large range of $a_{i}$, but BN-True events place much tighter constraints on $a_{i}$. For example, Case 3, which is the most favored, requires the initial binary $\left[\theta^{1} \mathrm{C}_{2}, \mathrm{BN}\right]$, i.e. two approximately equal-mass stars of $\sim 9 \mathrm{M}_{\odot}$, to have originally had $a_{i} \simeq 8 \pm 2 \mathrm{AU}$. Note that depending on the case, different ranges of $a_{i}$ contribute towards creating systems similar to the observed $\mathrm{BN}-\theta^{1} \mathrm{C}$ system. Moreover, note that the $a_{i}$ ranges where the BN-True events occur via the three Cases 1 - 3, ejection of $\mathrm{BN}$ is the most likely outcome among all other possible outcomes (except of course weak fly-bys; Figure 2). This gives us further confidence in the scenario that the $\mathrm{BN}-\theta^{1} \mathrm{C}$ system has been created via a strong binary-single interaction involving these three stars.

Throughout this study we have used the central values of the estimated masses for the three stars. Dynamically there should be no qualitative difference in the outcomes if the masses are changed within the mass errors. However, note that the masses of $\theta^{1} \mathrm{C}_{2}$ and $\mathrm{BN}$ are comparable. In fact the error ranges actually overlap. Due to the comparable masses, dynamically there is only a small difference between ejection of $\mathrm{BN}$ and ejection of $\theta^{1} \mathrm{C}_{2}$, as named here. This is reflected in our results to some degree. Cases 1 and 2 contribute towards creating the $\mathrm{BN}-\theta^{1} \mathrm{C}$ system over very similar $a_{i}$ ranges. Their contributions are also comparable. The small differences in the $a_{i}$ range and $\Sigma_{\mathrm{BN}-\text { True }}$ come from the small difference between $\theta^{1} \mathrm{C}_{2}$ and BN's assumed masses and also to some extent the scenario, a fly-by being more likely compared to an exchange if all else is kept unchanged. In fact, similar results will be recovered if $\mathrm{BN}$ and $\theta^{1} \mathrm{C}_{2}$ are interchanged among themselves. However, in that case, the definitions of Cases 1 and 2 will also be interchanged. 


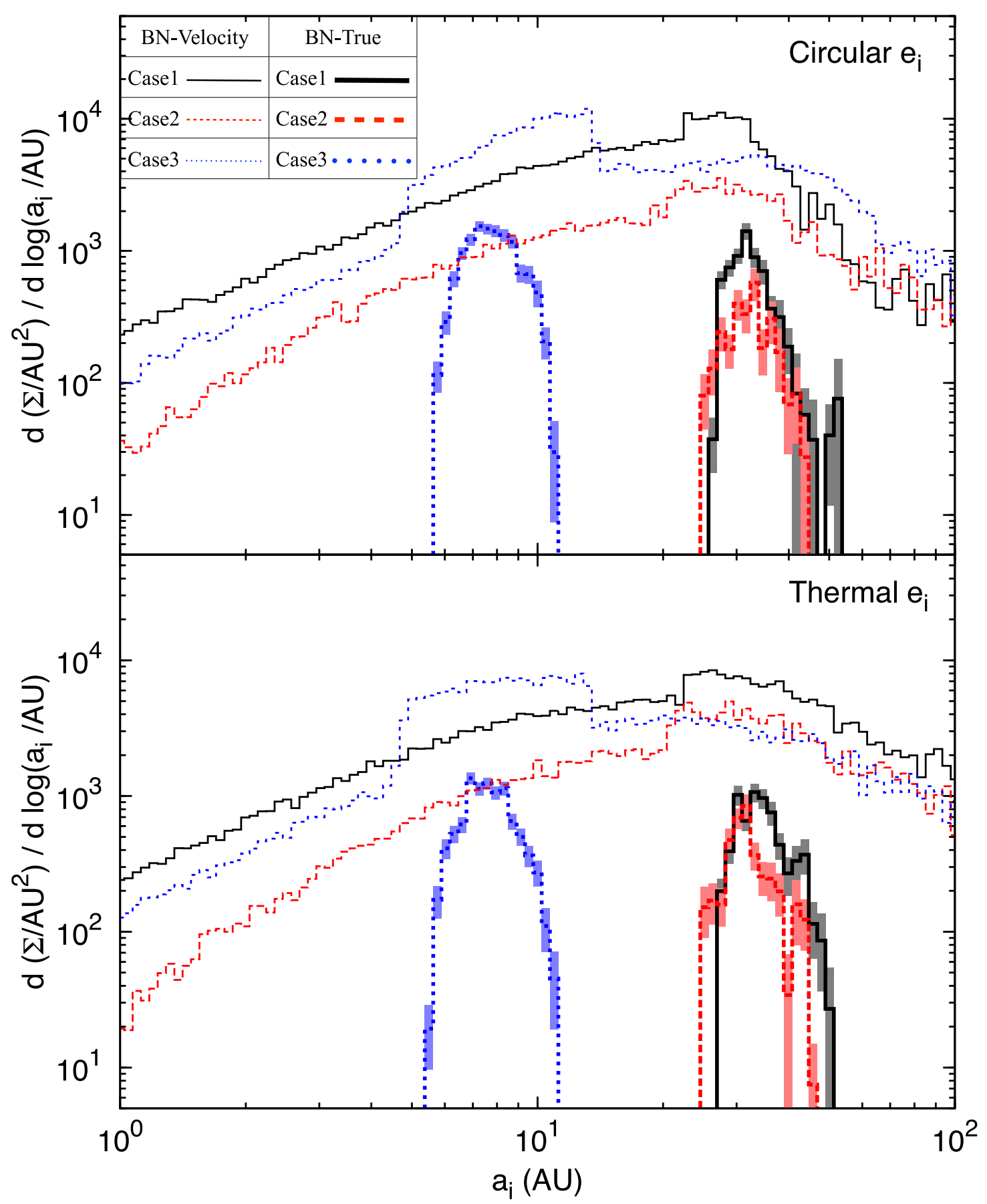

FIG. 7.- Same as Figure 3, but for $\Sigma$ as a function of the initial semimajor axis, $a_{i}$, of the binary. We find that all three cases and $e_{i}$-distributions can contribute to the production of the observed system over different $a_{i}$-ranges, although Case 3 has a relatively higher integrated $\Sigma$ for both $e_{i}$-distributions and selects from a quite distinct range of $a_{i} \simeq 8 \pm 2 \mathrm{AU}$.

\subsection{Orientation of the Orbital Plane of $\theta^{1} \mathrm{C}$ Binary Relative to the Direction of BN's Motion}

One additional variable in the dynamical ejection problem is the angle $\alpha$ between the angular momentum vector, $\vec{L}_{\theta^{1} \mathrm{C}}$, of the $\theta^{1} \mathrm{C}$ binary and the $3 D$ velocity vector of $\mathrm{BN}, \vec{v}_{\mathrm{BN}}$. Figure 8 shows the distribution of $\Sigma_{\mathrm{BN}-\text { True }}$ for all BN-True events for any $a_{i}$ as a function of $\alpha$ for all Cases 1-3 and all $e_{i}$ distributions. The distributions of $\alpha$ are quite broad for Cases 1 and 2. In comparison, for Case 3 there is a strong peak near $\alpha=90^{\circ}$.

To calculate the observed value of $\alpha$ for comparison with the predictions of our numerical results we adopt different measured values in existing literature. Orientation of $\vec{L}_{\theta^{1} \mathrm{C}}$ is calculated using data given in Kraus et al. (2009). The LSR velocity of BN is obtained from Scoville et al. (1983). There are two independent proper- 


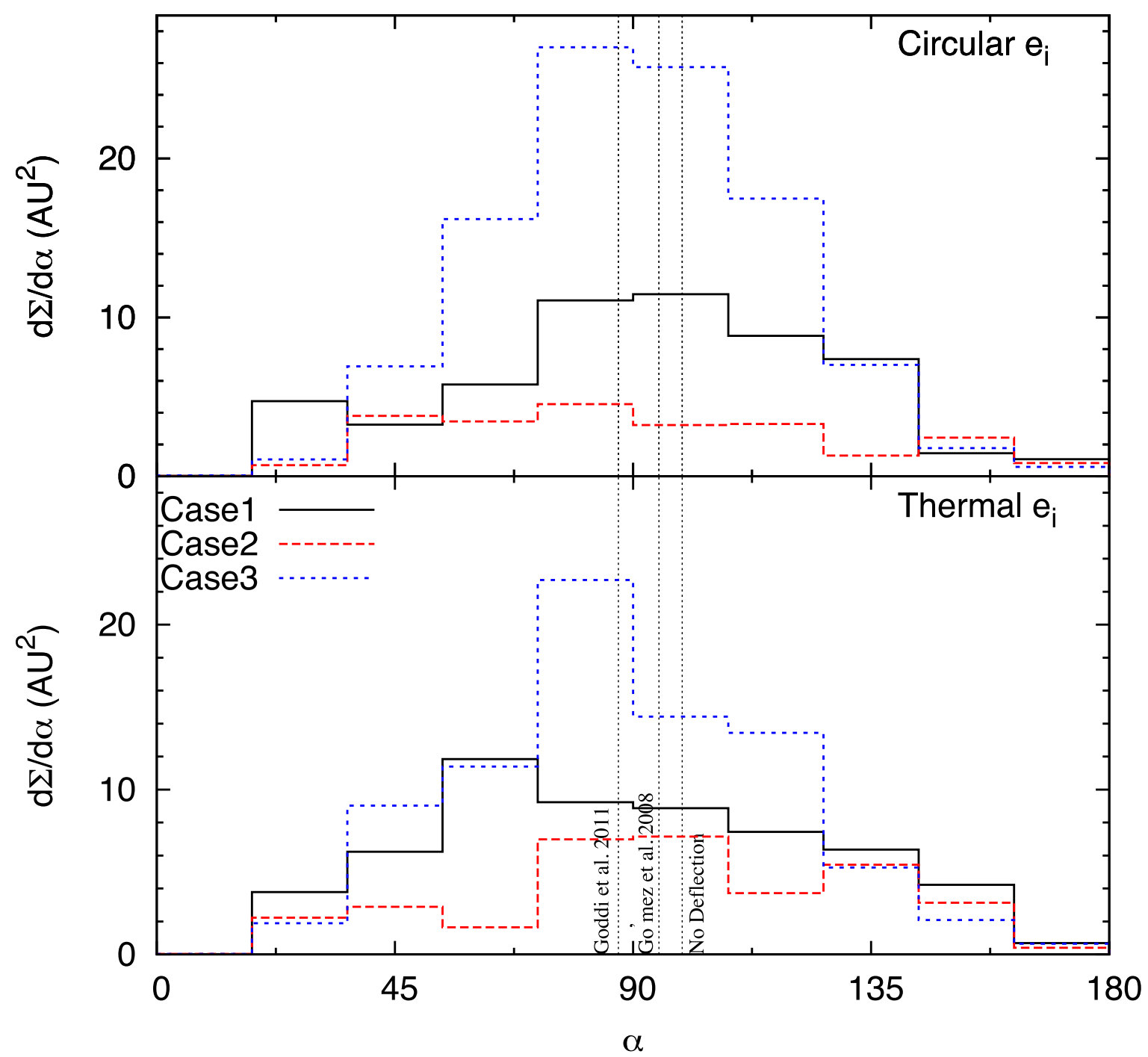

FIG. 8.- Cross-section, $\Sigma$, vs the angle $(\alpha)$ between the $3 D$ velocity of the runaway BN star $\vec{v}_{\mathrm{BN}}$ and the angular momentum vector $\vec{L}_{\theta^{1} \mathrm{C}}$ of the $\theta^{1} \mathrm{C}$ binary. The solid (black), dashed (red), and dotted (blue) lines denote Cases 1,2 , and 3, respectively, for BN-True events (distributions of BN-Velocity events are very similar). The top and bottom panels are for the Circular and Thermal $e_{i}$ distributions, respectively. The vertical dotted (black) lines show three values of $\alpha$ for the observed $\mathrm{BN}-\theta^{1} \mathrm{C}$ system where the values are calculated using $\vec{v}_{\mathrm{BN}}$ measurements by Goddi et al. (2011); Gómez et al. (2008) and assuming no deflection of BN after its ejection from the $\theta^{1} \mathrm{C}$ binary. Orientation of the $\theta^{1} \mathrm{C}$ binary is obtained from Kraus et al. (2009). The predicted distributions from our simulations are consistent with the estimated values of $\alpha$ for the observed $\mathrm{BN}-\theta^{1} \mathrm{C}$ system.

motion measurements for BN. Adopting the values given in Gómez et al. (2008) we find $\alpha=95^{\circ}$. Adopting the values given in Goddi et al. (2011) we find a slightly different value of $\alpha=87^{\circ}$. If the direction of BN's motion in the sky-plane is obtained by simply joining the expected position of the binary-single encounter and BN's current position, then $\alpha=99^{\circ}$. (this may be a more accurate value, since we expect $\mathrm{BN}$ to have suffered a recent change in its proper motion vector via interaction with source $I$, see below) Note all of the above values of $\alpha$ for the observed $\mathrm{BN}-\theta^{1} \mathrm{C}$ system are consistent with the predicted distribution of $\alpha$ from our numerical experiments, and give some support for the ejection having resulted via Case 3 .

4. TESTS FOR THE EJECTION SCENARIO OF BN FROM $\theta^{1} \mathrm{C}$ AND PROBABILITY OF CHANCE AGREEMENT
The system which ejected BN must be located along BN's past trajectory and have a total mass $\gtrsim 2 m_{\mathrm{BN}}$. These conditions are potentially satisfied for $\theta^{1} \mathrm{C}$, the 3 other Trapezium stars $\theta^{1} \mathrm{~A}, \theta^{1} \mathrm{~B}, \theta^{1} \mathrm{D}$, another ONC member $\theta^{2} A$, and probably for source $I$ (assuming it is the main source of luminosity in the KL nebula). Indeed, a number of authors have argued BN was launched from source I (Bally \& Zinnecker 2005; Gómez et al. 2008). However, as we now discuss, there are 6 independent properties of $\theta^{1} \mathrm{C}$ that have the values expected if it were the binary left behind after ejecting $\mathrm{BN}$ ( 7 if we assume ejection via Case 3 and include the angle $\alpha$ between the angular momentum vector, $\vec{L}_{\theta^{1} \mathrm{C}}$, of the $\theta^{1} \mathrm{C}$ binary and the $3 D$ velocity vector of $\left.\mathrm{BN}, \vec{v}_{\mathrm{BN}}\right)$. To consider the likelihood that all of these properties of the $\mathrm{BN}-\theta^{1} \mathrm{C}$ system are as observed by chance, we take that to be our null 
hypothesis. Given that BN has the runaway velocity, for each of these properties we assign a probability that $\theta^{1} \mathrm{C}$ has its values by chance to finally calculate the composite probability of chance agreement of $\theta^{1} \mathrm{C}^{\prime} \mathrm{s}$ properties with those expected from a binary-single scattering scenario. We discuss these properties and our estimates of the chance-agreement probabilities below. These probabilities are summarized in Table 1 along with the values predicted by the binary-single ejection scenario, and the observed values of the $\mathrm{BN}-\theta^{1} \mathrm{C}$ properties.

(1) ONC-Frame Proper Motion in Declination $\left(\mu_{\delta, \text { ONC }}\left(\theta^{1} \mathrm{C}\right)\right)$ : If $\theta^{1} \mathrm{C}$ ejected $\mathrm{BN}$, then, in the frame of the center of mass of the pre-ejection triple, the predicted value of $\mu_{\delta, \mathrm{T}}\left(\theta^{1} \mathrm{C}\right)=-\left(m_{\mathrm{BN}} / m_{\theta^{1} \mathrm{C}}\right) \mu_{\delta, \mathrm{T}}(\mathrm{BN}) \rightarrow$ $-\left(\left[9.3 \pm 2.0 \mathrm{M}_{\odot}\right] /\left[47 \pm 4 \mathrm{M}_{\odot}\right]\right) 11.7 \pm 1.3 \mathrm{mas} \mathrm{yr}^{-1} \rightarrow$

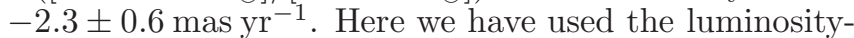
based mass estimate for BN (Tan 2004) and the proper motion measurements of Gómez et al. (2008) for BN, including a 0.70 mas/yr uncertainty of the motion in declination of the pre-ejection triple with respect to the $\mathrm{ONC}$ frame. The predicted value of the ONC-frame motion of $\theta^{1} \mathrm{C}$ is then $\mu_{\delta, \mathrm{ONC}}\left(\theta^{1} \mathrm{C}\right)[$ predicted $]=-2.3 \pm 0.9 \mathrm{mas} \mathrm{yr}^{-1}$, with the error increasing again because of the uncertain motion of the pre-ejection triple ${ }^{3}$. The observed value (van Altena et al. 1988) is $\mu_{\delta, \text { ONC }}\left(\theta^{1} C\right)$ [observed] $=-1.8 \pm 0.2$ mas yr $^{-1}$. Given the observed (van Altena et al. 1988) 1D proper motion dispersion of the bright ONC stars of 0.7 mas/yr, the probability for $\theta^{1} \mathrm{C}$ to be in the predicted range is 0.023 . Indeed, van Altena et al. (1988) already noted that $\theta^{1} \mathrm{C}$ has an abnormally large proper motion.

(2) ONC-Frame Proper Motion in Right Ascension $\left(\mu_{\alpha, \text { ONC }}\left(\theta^{1} \mathrm{C}\right) \cos \delta\right)$ : Similarly, in the frame of the center of mass of the pre-ejection triple: $\mu_{\alpha, \mathrm{T}}\left(\theta^{1} \mathrm{C}\right) \cos \delta=$ $-\left(m_{\mathrm{BN}} / m_{\theta^{1} \mathrm{C}}\right) \mu_{\alpha, \mathrm{T}}(\mathrm{BN}) \cos \delta \rightarrow-\left(\left[9.3 \pm 2.0 \mathrm{M}_{\odot}\right] /[47 \pm\right.$ $\left.\left.4 \mathrm{M}_{\odot}\right]\right)(-6.1 \pm 1.2)$ mas yr$^{-1} \rightarrow+1.21 \pm 0.36$ mas yr $^{-1}$. The predicted value of the ONC-frame motion of $\theta^{1} \mathrm{C}$ is then $\mu_{\alpha, \mathrm{ONC}}\left(\theta^{1} \mathrm{C}\right) \cos \delta[$ predicted] $=+1.2 \pm$

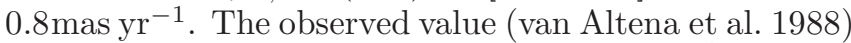
is $\mu_{\alpha, \theta^{1} \mathrm{C}} \cos \delta$ [observed] $=+1.4 \pm 0.2 \mathrm{mas} \mathrm{yr}^{-1}$ and the probability that $\theta^{1} \mathrm{C}$ is in the predicted range by chance is 0.27 .

(3) ONC-Frame Radial Velocity $\left(v_{\mathrm{r}, \mathrm{ONC}}\left(\theta^{1} \mathrm{C}\right)\right)$ : Similarly, the radial recoil in the frame of the pre-ejection triple should satisfy: $v_{\mathrm{r}, \mathrm{T}}\left(\theta^{1} \mathrm{C}\right)=$ $-\left(m_{\mathrm{BN}} / m_{\theta^{1} \mathrm{C}}\right) v_{\mathrm{r}, \mathrm{T}}(\mathrm{BN}) \rightarrow-\left(\left[9.3 \pm 2.0 \mathrm{M}_{\odot}\right] /[47 \pm\right.$ $\left.\left.4 \mathrm{M}_{\odot}\right]\right)\left(+13 \pm 1.8 \mathrm{~km} \mathrm{~s}^{-1}\right) \rightarrow-2.57 \pm 0.69 \mathrm{~km} \mathrm{~s}^{-1}$. The predicted value of the ONC-frame motion of $\theta^{1} \mathrm{C}$ is then $v_{\mathrm{r}, \mathrm{ONC}}\left(\theta^{1} \mathrm{C}\right)\left[\right.$ predicted] $=-2.6 \pm 1.6 \mathrm{~km} \mathrm{~s}^{-1}$, with the error range dominated by the assumption that the pre-ejection triple had a motion similar to the other bright ONC stars (van Altena et al. 1988) with $\sigma_{1 \mathrm{D}}=1.4 \mathrm{~km} \mathrm{~s}^{-1} \cdot \theta^{1} \mathrm{C}$ has an observed heliocentric velocity (Kraus et al. 2009) of $+23.6 \mathrm{~km} \mathrm{~s}^{-1}$ i.e. an LSR velocity of $5.5 \mathrm{~km} \mathrm{~s}^{-1}$, i.e. an $\mathrm{ONC}$ frame velocity of $v_{\mathrm{r}, \text { ONC }}\left(\theta^{1} \mathrm{C}\right)\left[\right.$ observed] $=-2.5 \mathrm{~km} \mathrm{~s}^{-1}$. For a Gaussian distribution with $\sigma_{1 \mathrm{D}}=1.4 \mathrm{~km} \mathrm{~s}^{-1}$, i.e. based on the proper motion dispersion of bright stars (van Altena

\footnotetext{
3 Note that here and for the other $\theta^{1} \mathrm{C}$ properties we have adopted $1 \sigma$ errors when possible, but not all physical properties have well-defined uncertainties: e.g., the model dependent $m_{\mathrm{BN}}$ estimate given its observed luminosity.
}

et al. 1988) the probability of being in the predicted velocity range by chance is 0.24 .

(4) Mass of Secondary $\left(m_{\theta^{1} \mathrm{C}_{2}}\right)$ : Given a $\theta^{1} \mathrm{C}$ primary mass of $38.2 \mathrm{M}_{\odot}$, what is the probability of having a secondary star with mass $\gtrsim m_{\mathrm{BN}}$ ? We estimate this probability using the low value $\left(m_{\mathrm{BN}}=7.3 \mathrm{M}_{\odot}\right)$ of the luminosity-based mass estimate for BN of $9.3 \pm 2.0 \mathrm{M}_{\odot}$. If the secondary star is drawn from a Salpeter powerlaw mass function, $d F / d m_{*} \propto m_{*}^{-2.35}$, where $F$ is the fraction of the stellar population, with maximum mass equal to the primary mass and lower mass limit equal to $1.0 \mathrm{M}_{\odot}$ (a relatively top-heavy IMF, with average mass of $2.8 M_{\odot}$, compared to the global ONC IMF, which has a broad peak around $0.5 M_{\odot}$, e.g., Muench et al. 2002), then the probability of having a secondary with mass $>7.3 \mathrm{M}_{\odot}$ is 0.061 . Hillenbrand \& Hartmann (1998) find evidence for mass segregation in the IMF (of primary stars) in the center of the ONC, with average stellar mass reaching peak values of $\sim 1.3-2 M_{\odot}$ in the vicinity of $\theta^{1} \mathrm{C}$ (excluding $\theta^{1} \mathrm{C}$ from the average). If we raise the lower limit of the above Salpeter IMF to $2 \mathrm{M}_{\odot}$ (i.e. an average mass of $5.1 M_{\odot}$ ), this raises the probability of obtaining a sufficiently massive secondary to 0.16 and we adopt this number as a conservative estimate. We note that none of the other Trapezium stars has a secondary mass that satisfies this condition (Tan 2004).

(5) Ratio of Ejection Kinetic Energy to Binary Total Energy $\left(E_{\text {ratio }}\left(B N-\theta^{1} \mathrm{C}\right)\right)$ : From our numerical experiments we find that in order for $\theta^{1} \mathrm{C}$ to have ejected $\mathrm{BN}$ at its observed velocity, $0.23<E_{\text {ratio }}[$ predicted] $<0.72$. This range contains about $70 \%$ of the BN-Velocity events. Recall, $E_{\text {ratio }}[$ observed $]=0.49 \pm 0.22$. Given the primary and secondary masses of $\theta^{1} \mathrm{C}$, what is the probability the total energy of the binary, $E_{\text {binary }}$, falls in the predicted range of 1.4 to $4.3 \times T_{\text {ejection, }}$ i.e. $(1.1-3.5) \times 10^{47} \mathrm{erg}$, simply by chance? This corresponds to a range of semi-major axes of 8.5 to $27 \mathrm{AU}$. If the distribution of $a$ follows $d F_{b} / d \log a=$ constant (Heggie \& Hut 2003) from 0.1 to $6300 \mathrm{AU}$ (see §2), then the probability that the $\theta^{1} \mathrm{C}$ binary falls in this range is 0.10 . If we assume ejection occurred via Case 3 , then $0.44<E_{\text {ratio }}$ [predicted] $<0.83$, corresponding to a range of semi-major axes of 16 to $30 \mathrm{AU}$ and a probability of chance agreement of 0.057 . The upper limit of allowed $a$ may be smaller than $6300 \mathrm{AU}$ for conditions in the central regions of the ONC. For a stellar density of $\sim 10^{4} \mathrm{pc}^{-1}$, the average separation is $6000 \mathrm{AU}$. Most of these stars will have masses lower than $\theta^{1} \mathrm{C}_{2}$ or $\mathrm{BN}$. Hence it is not likely that these lower mass stars will disrupt the relatively more massive $\mathrm{BN}$ or $\theta^{1} \mathrm{C}_{2}$ stars from being in a binary with $\theta^{1} \mathrm{C}_{1}$. If we adopt an upper limit smaller by a factor of 2 , i.e. $3000 \mathrm{AU}$, then the probabilities of the $\theta^{1} \mathrm{C}$ binary falling in the above expected ranges by chance rises by just $7 \%$.

(6) Eccentricity $\left(e\left(\theta^{1} \mathrm{C}\right)\right)$ : Our numerical experiments (see Figure 2) show that a very broad range of eccentricities is expected for the $\theta^{1} \mathrm{C}$ binary if it has ejected $\mathrm{BN}$ at the observed velocity (the average value for all the $\mathrm{BN}-\mathrm{Velocity}$ outcomes is $e=0.60$ with a $1 \sigma$ range from 0.34 to 0.86 ; for Case 3 this range is from 0.34 to $0.82)$. The observed value of $e=0.592 \pm 0.07$ is consistent with these expectations, especially being close to the peak of the distribution resulting from Case 3 (Figure 


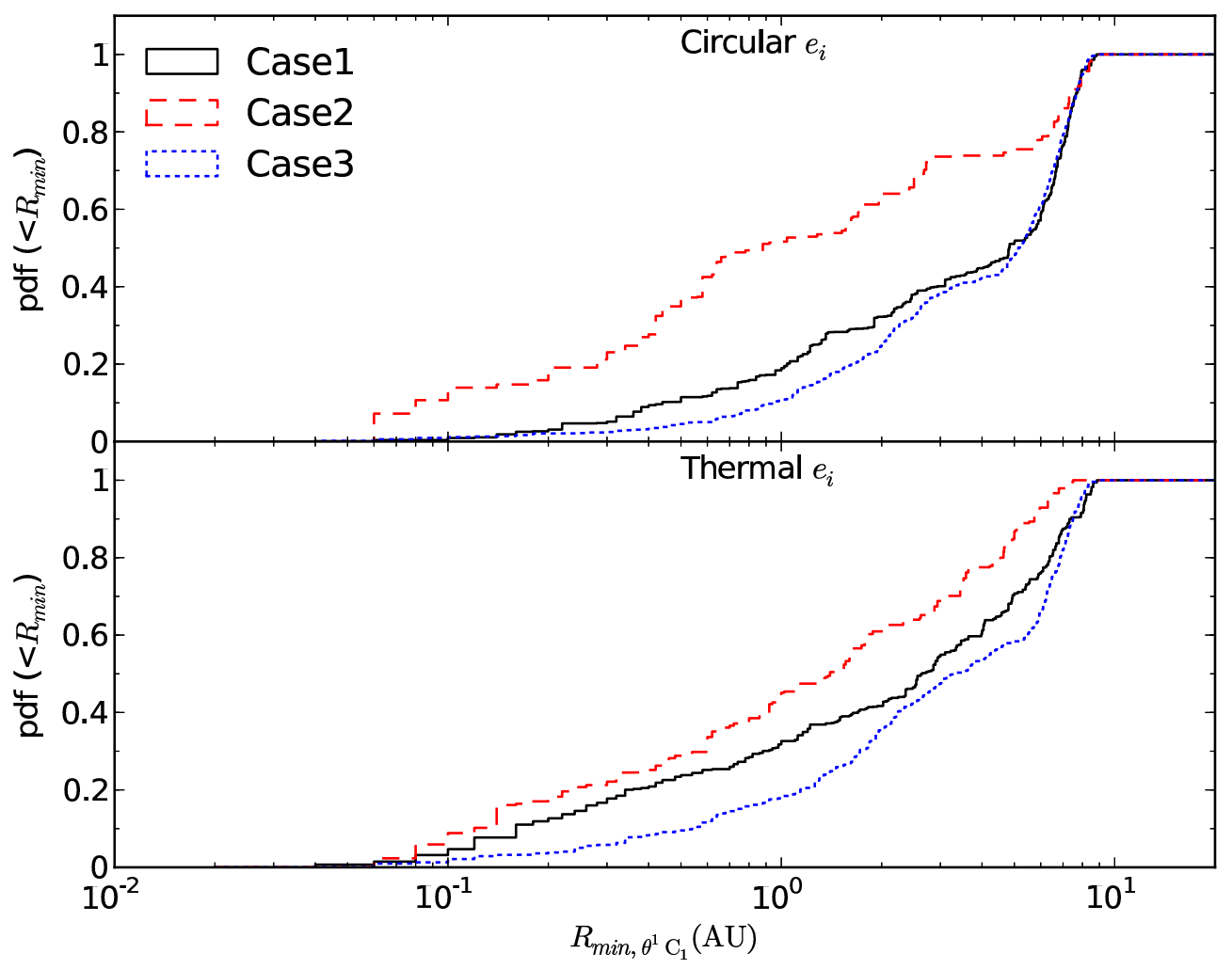

FIG. 9.- Cumulative histogram weighted by respective cross-sections for the closest approach of either $\mathrm{BN}$ or $\theta^{1} \mathrm{C}_{2}$ to $\theta^{1} \mathrm{C}_{1}\left(R_{m i n, \theta^{1} \mathrm{C}_{1}}\right)$ during the binary-single interactions for all cases and $e_{i}$-distributions for BN-True events. For all cases a significant fraction of interactions results in $R_{m i n, \theta^{1} \mathrm{C}_{1}} \gtrsim 5 \mathrm{AU}$. Especially, for Case 3 and Circular $e_{i}$-distribution, probability for $R_{\min , \theta^{1} \mathrm{C}_{1}} \gtrsim 5 \mathrm{AU}$ is about $60 \%$.

5). However, to assess the probability of this agreement by chance we need to know the eccentricity distribution of ONC binaries, especially for massive stars. Unfortunately there are few observational constraints on this eccentricity distribution. If the binaries have existed long enough to suffer many interactions, then a thermal distribution, $d F_{b} / d e=2 e$, is expected, which is weighted towards high eccentricities. A binary drawn from a thermal distribution has a 0.62 chance to be in the range $0.34<e<0.86$ (0.56 to be in the range $0.34<e<0.82$ for Case 3). Of course, if the actual distribution of $e$ of ONC binaries is close to circular $(e \simeq 0)$, then the probability of chance agreement for the eccentricity of $\theta^{1} \mathrm{C}$ with the value expected from $\mathrm{BN}$ ejection would be very small. To be conservative, we adopt the probability of 0.62 implied by a thermal distribution of eccentricities.

(7) Angle between the direction of $B N$ 's motion and the angular momentum of $\theta^{1} \mathrm{C}$ binary $(\alpha)$ : From our numerical experiments we find that for BN-Velocity events the angle $\alpha$ between $\hat{L}_{\theta^{1} \mathrm{C}}$ and $\hat{v}_{\mathrm{BN}}$ should satisfy $54^{\circ}<\alpha<126^{\circ}$. This range contains about $70 \%$ of all BN-True events. For Case 3 , the range is $58^{\circ}<\alpha<122^{\circ}$. The observed value of $\alpha$ for the $\mathrm{BN}-\theta^{1} \mathrm{C}$ system is $\sim 90^{\circ}$ and thus contained within this range. If $\theta^{1} \mathrm{C}$ and $\mathrm{BN}$ were unrelated, then the distribution of $\alpha$ should be $0.5 \sin \alpha$ over the range $0^{\circ}$ to $180^{\circ}$. Hence the probability for chance occurrence for $\alpha$ to be within the above range is
0.59 (0.53 for Case 3).

Combining the above individual probabilities and assuming, reasonably, that these properties are mutually independent, we find that the total probability of chance agreement of all 7 properties is small, $\epsilon=0.023 \times 0.27 \times$ $0.24 \times 0.16 \times 0.10 \times 0.62 \times 0.59=8.7 \times 10^{-6}$. If we assume ejection happened via Case 3 , which affects the last three probabilities, we obtain a total probability of chance agreement of $\epsilon=4.0 \times 10^{-6}$. The excellent agreement between the observed $\mathrm{BN}-\theta^{1} \mathrm{C}$-system properties and the predicted final properties from our numerical simulations (§3) together with the low chance agreement probability $(\epsilon)$ of all independent observed properties of the system strongly supports our proposed scenario that the observed system resulted from a strong binary-single ejection event involving $\theta^{1} \mathrm{C}_{1}, \theta^{1} \mathrm{C}_{2}$, and $\mathrm{BN}$ with a high probability of about $1-\epsilon \approx 0.99999$.

From the present day velocities and projected distance between the $\theta^{1} \mathrm{C}$ and $\mathrm{BN}$ this binary-single encounter must have happened about 4500 years ago at the location shown in Fig. 1. This scenario also explains the anomalously large proper motion of $\theta^{1} \mathrm{C}$ (van Altena et al. 1988), which will cause it to leave the central region of the cluster within $\sim 10^{5}$ years.

Based on a multi-frequency radial velocity analysis, Lehmann et al. (2010) have suggested $\theta^{1} \mathrm{C}$ may actually harbor an additional star of $1.0 \pm 0.16 \mathrm{M}_{\odot}$ in a close 
( $a=0.98 \mathrm{AU} ; P=61.5$ day), eccentric $(e=0.49)$ orbit around $\theta^{1} \mathrm{C}_{1}$. If during the proposed scattering event that ejected $\mathrm{BN}, \theta^{1} \mathrm{C}_{2}$ or $\mathrm{BN}$ came close to this inner region then one would expect likely ejection of the solar mass star. To examine the likelihood of such close interactions, in Figure 9 we show the cumulative histogram (weighted by cross-section) of closest approaches of either $\theta^{1} \mathrm{C}_{2}$ or $\mathrm{BN}$ to $\theta^{1} \mathrm{C}_{1}$ for all $\mathrm{BN}-\mathrm{True}$ events. For example for Case 3 with Circular $e_{i}, \sim 60 \%$ of the events happen with a closest approach that is $>5 \mathrm{AU}$. At these distances we would expect the solar mass star to remain relatively undisturbed in its orbit. Case 1 and especially Case 2 involve somewhat closer approaches, although both of them still have at least a 20\% contribution to events with closest approach $>5 \mathrm{AU}$. Future confirmation of the reality of the third star in the $\theta^{1} \mathrm{C}$ system may help us place further constraints on how BN was ejected from $\theta^{1} \mathrm{C}$.

\section{DISCUSSION AND SUMMARY}

We present the following argument in favor of our proposed scenario that $\mathrm{BN}$ was ejected by a binary-single interaction involving $\theta^{1} \mathrm{C}_{1}, \theta^{1} \mathrm{C}_{2}$, and $\mathrm{BN}$ in the past. $\mathrm{BN}$ is a runaway star (Plambeck et al. 1995; Tan 2004). It had to be launched by an interaction with a multiple system that has a primary mass greater than BN's mass along its past trajectory. The most massive binary in the $\mathrm{ONC}, \theta^{1} \mathrm{C}$, is a system satisfying this condition, but there are a few other candidates including, potentially, the massive protostar source I. To test whether $\theta^{1} \mathrm{C}$ binary ejected BN we consider 7 additional properties, namely recoil in 3 directions, sufficiently massive secondary, orbital binding energy, orbital eccentricity and angle between the orbital angular momentum vector and the direction of BN's velocity. Aided in part by a large and well-sampled suite of numerical simulations we show that all of these 7 observed properties of the $\mathrm{BN}-\theta^{1} \mathrm{C}$ system agree well with the properties predicted if $\mathrm{BN}$ was ejected by $\theta^{1} \mathrm{C}$. There are two and only two possibilities: 1) $\theta^{1} \mathrm{C}$ has all these properties by chance; 2) $\theta^{1} \mathrm{C}$ has acquired these properties naturally as a result of BN's ejection. We estimate the probability of chance agreement for all of the above properties to be low $\epsilon \lesssim 10^{-5}$. Hence, we conclude with about $(1-\epsilon) \approx\left(1-10^{-5}\right)$ probability that today's $\mathrm{BN}-\theta^{1} \mathrm{C}$ system was created via a binary-single interaction involving $\theta^{1} \mathrm{C}_{1}, \theta^{1} \mathrm{C}_{2}$, and $\mathrm{BN}$.

A summary of our numerical calculations is as follows. We performed $\sim 10^{7}$ numerical simulations that allowed us to properly sample the multidimensional parameter space effectively (§2). We used two different $e_{i}$ distributions as limiting cases, and 200 different $a_{i}$ values from the full range of possible $a_{i}$ in ONC from physical considerations for all three cases that can produce the observed $\mathrm{BN}-\theta^{1} \mathrm{C}$ system $(\S 2)$. We found that the predicted energies of the $\mathrm{BN}-\theta^{1} \mathrm{C}$ system agree well with the predictions from the scattering scenario (Figure 5) for both assumed $e_{i}$ distributions and all three cases. Furthermore, the predicted distribution of the angle $\alpha$ between BN's velocity vector $\vec{v}_{\mathrm{BN}}$ and $\theta^{1} \mathrm{C}$ 's angular momentum vector $\vec{L}_{\theta^{1} \mathrm{C}}$ was consistent with the observed value of $\alpha$ (Figure 8). Further calculations of cross-sections for the BN-Velocity and BN-True events constrained a range of initial binary semimajor axes for each Case $(1,2,3)$ that would produce the observed BN- $\theta^{1} \mathrm{C}$ system followed by a strong scattering (Figure 7 ). For these ranges of $a_{i}$ ejection of $\mathrm{BN}$ in general is the dominant outcome (apart from weak fly-bys which has a formally infinite cross-section; Figure 2). Our results indicate that all Cases can contribute to the production of the observed $\mathrm{BN}-\theta^{1} \mathrm{C}$ system. However, an interaction between an initial binary with members $\theta^{1} \mathrm{C}_{2}$ and $\mathrm{BN}$, and a single star $\theta^{1} \mathrm{C}_{1}$, our Case 3 (denoted by $\left.\left[\theta^{1} \mathrm{C}_{2}, \mathrm{BN}\right] \theta^{1} \mathrm{C}_{1} \longrightarrow\left[\theta^{1} \mathrm{C}_{1}, \theta^{1} \mathrm{C}_{2}\right] \mathrm{BN}\right)$, is favored by about a factor of 2 (Figure 6 ).

Ejection of $\mathrm{BN}$ from $\theta^{1} \mathrm{C}$ has several important implications for our understanding of massive star formation in the KL nebula, where a core of gas appears to be collapsing to form at least one massive star, thought to be detected in the radio as source $I$ (Figure 1 ).

If $\mathrm{BN}$ was ejected from $\theta^{1} \mathrm{C}$, then its passage near source $I$ within $\sim 0.5^{\prime \prime}$, i.e. a projected separation of $\sim 200$ AU, i.e. an expected physical separation (Gómez et al. 2008; Goddi et al. 2011) of 300 AU in the KL nebula is coincidental. Our estimated ejection point (Figure 1 ) is $50.1^{\prime \prime}$ from source $I$ 's present location, corresponding to 20,800 AU. The ONC-frame radial velocity is about half of the plane of sky velocity, implying $\mathrm{BN}$ also travelled 10,400 AU in the radial direction to reach source $I$, for a total distance of $23,200 \mathrm{AU}$. The probability to approach within $300 \mathrm{AU}$ of source $I$, ignoring gravitational focussing, is thus $\pi(300 / 23,200)^{2} / 4 \pi=4 \times 10^{-5}$. Gravitational focussing by $\sim 20 \mathrm{M}_{\odot}$ of total mass in and around source I (McKee \& Tan 2003; Wright et al. 1992) boosts the cross-section by $\sim 14 \%$, so the probability of approach is $\sim 5 \times 10^{-5}$. Thus the interaction of BN with KL is an improbable event. Of course, the chance of interaction of BN with any existing protostar, not necessarily source $I$, in the $\mathrm{ONC}$ is larger simply by a factor equal to the number of protostars in this volume around $\theta^{1} \mathrm{C}$. From X-ray observations (Grosso et al. 2005) there appear to be at least $\sim 10$ such objects even in just the local vicinity of KL, so the total probability of an interaction between BN and a protostar can be at least an order of magnitude higher, but still leaving it as being highly unlikely. We note that the probability that $\theta^{1} \mathrm{C}$ is masquerading as the system that ejected $\mathrm{BN}$ is even smaller than the probability of BN interacting with source $I$. Furthermore, the evidence for $\theta^{1} \mathrm{C}$ to have ejected $\mathrm{BN}$ is based on 7 independent lines of evidence and so survived several tests for falsification $(\S 4)$.

A close passage of BN with source $I$ will have deflected BN's motion by an angle

$7.5^{\circ}\left(m_{I} / 20 \mathrm{M}_{\odot}\right)(\mathrm{b} / 300 \mathrm{AU})^{-1}\left(\mathrm{v}_{\mathrm{BN}} / 30 \mathrm{~km} \mathrm{~s}^{-1}\right)^{-2} \quad$ towards source $I$, where $b$ is the initial impact parameter and $v_{\mathrm{BN}}$ is the velocity of $\mathrm{BN}$ relative to source $I$. We expect that a reasonably accurate estimate of the original ONC-frame position angle (P.A.) of BN's proper motion can be derived by considering the angle from the estimated position of the dynamical ejection from $\theta^{1} \mathrm{C}$ (the cross in Figure 1) and BN's current position, which is $-29^{\circ} .8$. The current observed P.A. of BN's ONC-frame proper motion is variously estimated to be $-27^{\circ} .5 \pm 4^{\circ}$ (Gómez et al. 2008) and $-18^{\circ} .8 \pm 4.6^{\circ}$ (Goddi et al. 2011). The average of these is $-23^{\circ} .1 \pm 3^{\circ}$, suggesting a projected deflection of $6^{\circ} .7 \pm \sim 3^{\circ}$ towards source $I$. The total true deflection may be expected to be $\sim \sqrt{2}$ larger, i.e. $\sim 9^{\circ} .5 \pm 3^{\circ}$, which is consistent 
TABLE 1

Current properties of $\theta^{1} \mathrm{C}$ That are Required if it Ejected BN

\begin{tabular}{|c|c|c|c|}
\hline Property of $\theta^{1} \mathrm{C}$ & Predicted Value & Observed Value $^{a}$ & $\begin{array}{c}\text { Probability of } \\
\text { Chance Agreement }\end{array}$ \\
\hline Proper Motion in Dec. ${ }^{\mathrm{b}}\left(\mu_{\delta, \mathrm{ONC}}\right)$ & $-2.3 \pm 0.9$ mas yr $^{-1}$ & $-1.8 \pm 0.2$ mas yr $^{-1}$ & 0.023 \\
\hline Proper Motion in R.A. ${ }^{\mathrm{b}}\left(\mu_{\alpha}, \mathrm{ONC} \cos \delta\right)$ & $+1.2 \pm 0.8$ mas yr $^{-1}$ & $+1.4 \pm 0.2 \operatorname{mas~yr}^{-1}$ & 0.27 \\
\hline Radial Velocity $\mathrm{b}\left(v_{\mathrm{r}, \mathrm{ONC}}\right)$ & $-2.6 \pm 1.6 \mathrm{~km} \mathrm{~s}^{-1}$ & $-2.5 \mathrm{~km} \mathrm{~s}^{-1}$ & 0.24 \\
\hline Mass of Secondary $\left(m_{\theta^{1} \mathrm{C}_{2}}\right)$ & $7.3-38.2 \mathrm{M} \odot$ & $8.8 \pm 1.7 \mathrm{M}_{\odot}$ & $0.16^{\mathrm{c}}$ \\
\hline Eject. KE to Binary Total E $\left(E_{\text {ratio }}\right)$ & $0.23-0.72$ & $0.49 \pm 0.22$ & $0.10[0.057]^{\mathrm{d}}$ \\
\hline Eccentricity $(e)$ & $0.34-0.86$ & $0.592 \pm 0.07$ & $0.62^{\mathrm{e}}[0.56]^{\mathrm{d}}$ \\
\hline Angle between $\hat{L}_{\theta^{1} \mathrm{C}} \& \hat{v}_{\mathrm{BN}}(\alpha)$ & $54^{\circ}-126^{\circ}$ & $\sim 90^{\circ}$ & $0.59[0.53]^{\mathrm{d}}$ \\
\hline Combination of 7 Independent Properties & & & $8.7 \times 10^{-6}\left[4.0 \times 10^{-6}\right]^{\mathrm{d}}$ \\
\hline
\end{tabular}

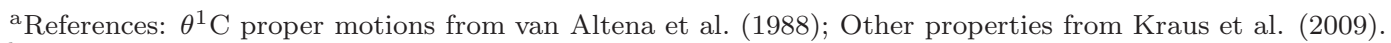

bONC-frame

${ }^{\mathrm{c}}$ Assumes secondary is drawn from a Salpeter initial mass function (IMF) with lower mass limit of $2 \mathrm{M} \odot . \mathrm{A}$ lower limit of $1 \mathrm{M}_{\odot}$ ( still a top-heavy IMF) would yield a probability of 0.061 .

dNumber in square brackets assumes ejection via Case 3.

e Assumes binary eccentricity is drawn from a thermal distribution, which is the most eccentric distribution that can be expected (requiring the cluster stars to have had a long enough time to interact). Thus this probability should be regarded as a conservative upper limit.
}

with our previous estimate.

Close passage of BN near the accretion disk of source $I$ about 500 years ago would induce tidal perturbations that enhance the effective viscosity of the disk and thus its accretion rate (Tan 2004). This can explain the enhanced, apparently explosive, outflow (Allen \& Burton 1993), which has observed timescales of $\sim 500-1000 \mathrm{yr}$, if the inner region of the disk with an orbital time $\lesssim 500$ yr has been significantly perturbed. This corresponds to disk radii of $r \lesssim 180\left(m_{I} / 20 \mathrm{M}_{\odot}\right)^{1 / 3} \mathrm{AU}$, which is consistent with the estimate of closest approach based on deflection of BN's trajectory.

The estimated current accretion rate to source $I$ is $\sim 3 \times 10^{-3} \mathrm{M}_{\odot} \mathrm{yr}^{-1}$ to a $10 \mathrm{M}_{\odot}$ protostar (and somewhat higher rates if the protostar is more massive) (Testi et al. 2010). This is about a factor of 10 higher than expected given the properties of the gas core (McKee \& Tan 2002, 2003). If constant over the last $1000 \mathrm{yr}$, this would imply a total accreted mass of about $3 \mathrm{M}_{\odot}$, which would be a significant fraction of the original accretion disk-mass around a $10-20 \mathrm{M}_{\odot}$ protostar, since the disk mass is expected to be limited to $\sim 30 \%-100 \%$ of the stellar mass by gravitational torques (Kratter et al. 2010). The mass launched by a magneto-hydrodynamic outflow during this time is expected to be $\approx 30 \%$ of this amount (Najita \& Shu 1994), i.e. about $1 \mathrm{M}_{\odot}$. This is consistent with the mass estimated (Chernoff et al. 1982) to be in the inner, "explosive" part of the outflow of about $3 \mathrm{M}_{\odot}$.

Our results suggest that the formation of a massive star in the KL nebula, i.e. source $I$, has been affected by an external perturbation of a runaway B star, BN, ejected from a different region of the cluster, i.e. by $\theta^{1} \mathrm{C}$. For BN to be launched so close to a forming massive protostar does appear to be an intrinsically unlikely event, but multiple pieces of independent evidence strongly support this scenario. It also explains source $I$ s anomalously high accretion rate and the unusual, apparently "explosive" nature of the recent outflow from this source. It is possible that a significant fraction, up to $\sim 1 / 3$, of the accreted mass has been induced by this perturbation. In other respects, the Core Accretion model (McKee \& Tan 2002,2003 ) of massive star formation provides a reasonable description of the system, e.g., the presence of a massive core around the protostar and two wide-angle outflow cavities from which near-IR light emerges (Testi et al. 2010). The example of Orion BN-KL suggests that, occasionally, in crowded regions near the center of star clusters, the star formation model needs to be modified to account for tidal perturbations from external, passing stars.

Improved observational constraints on the properties of $\theta^{1} \mathrm{C}$ especially its binary properties and ONC-frame proper motion, and the mass and current proper motion of BN will place even more stringent tests on the proposed ejection scenario and also help improve the dynamical mass constraints on source $I$ from the deflection of BN.

We thank John Bally, Paola Caselli, Eric Ford, John Fregeau, Ciraco Goddi, Lincoln Greenhill, Stefan Kraus, Chris McKee, Francesco Palla, Hagai Perets, Dick Plambeck and Leonardo Testi for helpful discussions. We thank the anonymous referee for a detail review. JCT acknowledges support from NSF CAREER grant AST0645412; NASA Astrophysics Theory and Fundamental Physics grant ATP09-0094; NASA Astrophysics Data Analysis Program ADAP10-0110 and a Faculty Enhancement Opportunity grant from the University of Florida. SC acknowledges support from the Theory Postdoctoral Fellowship from UF Department of Astronomy and College of Liberal Arts and Sciences.

\section{REFERENCES}

Adams, F. C. 2010, ARA\&A, 48, 47

Allen, D. A. \& Burton, M. G. 1993, Nature, 363, 54
Bally, J., Cunningham, N. J., Moeckel, N., Burton, M. G., Smith, N., Frank, A., \& Nordlund, A. 2011, ApJ, 727, 113

Bally, J. \& Zinnecker, H. 2005, AJ, 129, 2281 
Bonnell, I. A., Bate, M. R., Clarke, C. J., \& Pringle, J. E. 2001, MNRAS, 323, 785

Bonnell, I. A., Bate, M. R., \& Zinnecker, H. 1998, MNRAS, 298, 93

Chernoff, D. F., McKee, C. F., \& Hollenbach, D. J. 1982, ApJ, 259, L97

Da Rio, N., Robberto, M., Soderblom, D. R., Panagia, N. Hillenbrand, L. A., Palla, F., \& Stassun, K. G. 2010, ApJ, 722, 1092

Eggleton, P. P., Tout, C. A., \& Fitchett, M. J. 1989, ApJ, 347, 998

Fưrész, G., Hartmann, L. W., Megeath, S. T., Szentgyorgyi, A. H., \& Hamden, E. T. 2008, ApJ, 676, 1109

Fregeau, J. M., Chatterjee, S., \& Rasio, F. A. 2006, ApJ, 640, 1086

Fregeau, J. M., Cheung, P., Portegies Zwart, S. F., \& Rasio, F. A. 2004, MNRAS, 352, 1

Fujii, M. S. \& Portegies Zwart, S. 2011, Science, 334, 1380

Gezari, D. Y., Backman, D. E., \& Werner, M. W. 1998, ApJ, 509, 283

Gies, D. R. \& Bolton, C. T. 1986, ApJS, 61, 419

Goddi, C., Humphreys, E. M. L., Greenhill, L. J., Chandler, C. J., \& Matthews, L. D. 2011, ApJ, 728, 15

Gómez, L., Rodríguez, L. F., Loinard, L., Lizano, S., Allen, C., Poveda, A., \& Menten, K. M. 2008, ApJ, 685, 333

Grosso, N., Feigelson, E. D., Getman, K. V., Townsley, L., Broos, P., Flaccomio, E., McCaughrean, M. J., Micela, G., Sciortino, S., Bally, J., Smith, N., Muench, A. A., Garmire, G. P., \& Palla, F. 2005, ApJS, 160, 530

Gualandris, A., Portegies Zwart, S., \& Eggleton, P. P. 2004, MNRAS, 350, 615

Gvaramadze, V. V. \& Gualandris, A. 2011, MNRAS, 410, 304

Heggie, D. \& Hut, P. 2003, The Gravitational Million-Body Problem: A Multidisciplinary Approach to Star Cluster Dynamics (Cambridge University Press, 2003)

Hillenbrand, L. A. \& Hartmann, L. W. 1998, ApJ, 492, 540

Hut, P. \& Bahcall, J. N. 1983, ApJ, 268, 319

Jones, B. F. \& Walker, M. F. 1988, AJ, 95, 1755

Kratter, K. M., Matzner, C. D., Krumholz, M. R., \& Klein, R. I. 2010, ApJ, 708, 1585
Kraus, S., Weigelt, G., Balega, Y. Y., Docobo, J. A., Hofmann, K.-H., Preibisch, T., Schertl, D., Tamazian, V. S., Driebe, T. Ohnaka, K., Petrov, R., Schöller, M., \& Smith, M. 2009, A\&A, 497, 195

Lehmann, H., Vitrichenko, E., Bychkov, V., Bychkova, L., \& Klochkova, V. 2010, A\&A, 514, A34

McCaughrean, M., Zinnecker, H., Andersen, M., Meeus, G., \& Lodieu, N. 2002, The Messenger, 109, 28

McKee, C. F. \& Tan, J. C. 2002, Nature, 416, 59

-. 2003, ApJ, 585, 850

McMillan, S. L. W. \& Hut, P. 1996, ApJ, 467, 348

Menten, K. M. \& Reid, M. J. 1995, ApJ, 445, L157

Menten, K. M., Reid, M. J., Forbrich, J., \& Brunthaler, A. 2007, A\&A, 474, 515

Muench, A. A., Lada, E. A., Lada, C. J., \& Alves, J. 2002, ApJ, 573,366

Najita, J. R. \& Shu, F. H. 1994, ApJ, 429, 808

Plambeck, R. L., Wright, M. C. H., Mundy, L. G., \& Looney, L. W. 1995, ApJ, 455, L189

Poveda, A., Ruiz, J., \& Allen, C. 1967, Boletin de los Observatorios Tonantzintla y Tacubaya, 4,86

Scoville, N., Kleinmann, S. G., Hall, D. N. B., \& Ridgway, S. T. 1983, ApJ, 275, 201

Tachibana, S. \& Huss, G. R. 2003, ApJ, 588, L41

Tan, J. C. 2004, ApJ, 607, L47

—. 2008, ArXiv e-prints, arXiv:0807.3771v2 [astro-ph]

Testi, L., Tan, J. C., \& Palla, F. 2010, A\&A, 522, A44

van Altena, W. F., Lee, J. T., Lee, J.-F., Lu, P. K., \& Upgren, A. R. 1988, AJ, 95, 1744

Wang, P., Li, Z.-Y., Abel, T., \& Nakamura, F. 2010, ApJ, 709, 27

Wright, M., Sandell, G., Wilner, D. J., \& Plambeck, R. L. 1992, ApJ, 393, 225

Zwicky, F. 1957, Morphological astronomy, ed. Zwicky, F. 